\title{
Fuzzy Neighborhood Operators based on Fuzzy Coverings
}

\author{
Lynn D'eer ${ }^{\mathrm{a}, *}$, Chris Cornelis ${ }^{\mathrm{a}, \mathrm{b}}$, Lluís Godo ${ }^{\mathrm{c}}$ \\ ${ }^{a}$ Department of Applied Mathematics, Computer Science and Statistics, Ghent University, Belgium. \\ ${ }^{b}$ Department of Computer Science and Artificial Intelligence, Research Center on Information and Communications Technology (CITIC-UGR), \\ University of Granada, Spain. \\ ${ }^{c}$ Artificial Intelligence Research Institute (IIIA), Spanish National Research Council (CSIC), Bellaterra, Spain.
}

\begin{abstract}
In many data mining processes, neighborhood operators play an important role as they are generalizations of equivalence classes which were used in the original rough set model of Pawlak. In this article, we introduce the notion of fuzzy neighborhood system of an object based on a given fuzzy covering, as well as the notion of the fuzzy minimal and maximal descriptions of an object. Moreover, we extend the definition of four covering-based neighborhood operators as well as six derived coverings discussed by Yao and Yao to the fuzzy setting. We combine these fuzzy neighborhood operators and fuzzy coverings and prove that only sixteen different fuzzy neighborhood operators are obtained. Moreover, we study the partial order relations between those sixteen operators.
\end{abstract}

Keywords: fuzzy coverings, fuzzy neighborhood operators, rough sets

\section{Introduction}

Rough sets were introduced by Pawlak as a methodology for data analysis based on the approximation of concepts in data tables. It handles uncertainty in information systems due to indiscernibility and incompleteness. In the original model of Pawlak [17], an equivalence relation $E$ is used to describe the indiscernibility among pairs of objects of a universe $U$ and to define an approximation space. On the other hand, it is well-known that an equivalence relation can be equivalently specified by a partition of the universe, thus an approximation space can also be formulated in terms of a partition. In [28], Yao and Yao observe that still another equivalent structure can be considered to define the same approximation space, the $\sigma$-algebra of subsets of the universe whose atoms are the equivalence classes and whose elements are unions of these equivalence classes. Yao and Yao refer to these three equivalent structures as the element-based, the granule-based and the subsystem-based definitions of Pawlak's model.

From an application perspective, working with equivalence relations is often a too strong assumption in order to obtain useful results. Therefore, Pawlak's model has been generalized along each of the three mentioned formulations. Indeed, by replacing the equivalence relation in the element-based definition by a general binary relation, or equivalently by a neighborhood operator, a first generalization is obtained [21, 23, 26, 27]. In this case, the binary relation or the neighborhood operator determines collections of sets which no longer form a partition of $U$. A second generalization is obtained when we replace, in the granule-based definition, a partition by a covering, i.e., by a collection of non-empty sets such that its union is equal to $U[18,27,31]$. Finally, a third kind of generalized models is obtained when in the subsystem-based definition we replace the $\sigma$-algebra of subsets by a pair of systems: a closure system over $U$, i.e., a family of subsets of $U$ that contains $U$ and is closed under set-intersection, and its dual system [28]. However, these different generalizations are no longer equivalent, yielding different generalized rough set models.

For instance, in [28], given a covering $\mathbb{C}$, it is shown how one can derive four neighborhood operators as well as six other coverings, two of which coincide. The resulting twenty-four neighborhood operators are analyzed in [6]

\footnotetext{
${ }^{*}$ Corresponding author Godo)

Email addresses: Lynn. Deer@UGent.be (Lynn D’eer), Chris.Cornelis@decsai.ugr.es (Chris Cornelis), godo@iiia.csic.es (Lluís
} 
and reduced to thirteen groups of neighborhood operators. Moreover, the Hasse diagram of the thirteen neighborhood operators is obtained. Six of the considered neighborhood operators result in new covering-based rough set approximation operators, which are included in the framework of [20]. Furthermore, the connection between different covering-based approximation operators and relation-based approximation operators is discussed.

Both element-based and granule-based rough set models have been extended to the fuzzy setting in order to deal with real-valued data $[4,5]$. In these extensions, a key concept is that of a fuzzy neighborhood operator. In the literature, fuzzy neighborhood operators are often used in the context of fuzzy topology, e.g., [12, 14-16], in order to describe concepts such as open and closed sets, and interior and closure operators. Interior and closure operators are closely related with the concept of approximation operators in data analysis, and here we focus on the concept of a fuzzy neighborhood operator from the perspective of fuzzy rough set theory, which is a hybridization of fuzzy set theory [30] and rough set theory [17]. The combination of both theories yields massive potential for information systems with real-valued data. The fuzzy neighborhood operators discussed in this article can be used to define fuzzy rough approximation operators [4, 24].

In the last couple of years, initial efforts have been done to extend covering-based rough set models to the fuzzy setting $[5,9,10,13,22]$. Continuing the fuzzification of rough sets models, we extend here the definitions of the twenty-four neighborhood operators of [28] to the fuzzy setting, and we discuss equalities and partial order relations between them, extending the analysis done in [6] for the crisp case.

The outline of the article is as follows. In Section 2, we discuss some preliminary results concerning crisp neighborhood operators based on crisp coverings. In Section 3, different fuzzy neighborhood operators based on a fuzzy covering are introduced. Moreover, the properties of the fuzzy neighborhood operators are discussed. Additionally in Section 4, six fuzzy coverings derived from one fuzzy covering are studied. In Section 5, we discuss equalities between different fuzzy neighborhood operators based on a finite fuzzy covering and in Section 6, partial order relations between them are identified in order to obtain the Hasse diagram. Finally, we state some conclusions and future work in Section 7.

\section{Preliminaries}

Throughout this paper we assume that the universe $U$ is a non-empty set. We start by discussing the relevant concepts in the crisp setting.

A neighborhood operator [28] on the universe $U$ is a mapping $N: U \rightarrow \mathscr{P}(U)$, where $\mathscr{P}(U)$ represents the collection of subsets of $U$. In general, we assume that a neighborhood operator $N$ is reflexive, i.e., $x \in N(x)$ for each $x \in U$. Moreover, a neighborhood operator is called symmetric if for all $x, y \in U$ it holds that $x \in N(y)$ if and only if $y \in N(x)$, and it is called transitive if for all $x, y \in U$ it holds that $x \in N(y) \Rightarrow N(x) \subseteq N(y)$.

Given a universe $U$ and a collection $\mathbb{C}=\left\{K_{i} \subseteq U: K_{i} \neq \emptyset, i \in I\right\}$ of non-empty subsets of $U$, with $I$ an index set, $\mathbb{C}$ is called a covering of $U$ if $\bigcup_{i \in I} K_{i}=U$ [32]. A covering $\mathbb{C}$ induces the following neighborhood system for $x \in U$ [28]:

$$
\mathscr{C}(\mathbb{C}, x)=\{K \in \mathbb{C}: x \in K\}
$$

In a neighborhood system $\mathscr{C}(\mathbb{C}, x)$, the minimal and maximal sets that contain the element $x \in U$ are particularly important. The set

$$
\operatorname{md}(\mathbb{C}, x)=\{K \in \mathscr{C}(\mathbb{C}, x):(\forall S \in \mathscr{C}(\mathbb{C}, x))(S \subseteq K \Rightarrow K=S)\}
$$

is called the minimal description [2] of $x$. On the other hand, the set

$$
\operatorname{MD}(\mathbb{C}, x)=\{K \in \mathscr{C}(\mathbb{C}, x):(\forall S \in \mathscr{C}(\mathbb{C}, x))(S \supseteq K \Rightarrow K=S)\}
$$

is called the maximal description [33] of $x$. The sets $\operatorname{md}(\mathbb{C}, x)$ and $\operatorname{MD}(\mathbb{C}, x)$ are also called the minimal-description and maximal-description neighborhood systems of $x$ [28]. The importance of the minimal and maximal description of $x$ is demonstrated by the following proposition:

Proposition 1. [28] Let $\mathbb{C}$ be a covering and $K \in \mathscr{C}(\mathbb{C}, x)$. Then there exist $K_{1} \in \operatorname{md}(\mathbb{C}, x)$ and $K_{2} \in \operatorname{MD}(\mathbb{C}, x)$ such that $K_{1} \subseteq K \subseteq K_{2}$. Moreover, for all $x \in U$ it holds that $\bigcap \operatorname{md}(\mathbb{C}, x)=\bigcap \mathscr{C}(\mathbb{C}, x)$ and $\bigcup \operatorname{MD}(\mathbb{C}, x)=\bigcup \mathscr{C}(\mathbb{C}, x)$. 
We have seen that given a covering $\mathbb{C}$ and an element $x \in U$, we can consider three neighborhood systems for $x$ : $\mathscr{C}(\mathbb{C}, x)$ itself and two extreme neighborhood systems $\operatorname{md}(\mathbb{C}, x)$ and $\operatorname{MD}(\mathbb{C}, x)$. Furthermore, we can apply two extreme operations on these neighborhood systems by taking the intersection and the union of their elements. In this way, Yao and Yao [28] constructed the following four neighborhood operators based on the covering $\mathbb{C}$ :

1. $N_{1}^{\mathbb{C}}(x)=\bigcap\{K \in \mathbb{C}: K \in \operatorname{md}(\mathbb{C}, x)\}=\bigcap\{K \in \mathbb{C}: K \in \mathscr{C}(\mathbb{C}, x)\}$,

2. $N_{2}^{\mathbb{C}}(x)=\bigcup\{K \in \mathbb{C}: K \in \operatorname{md}(\mathbb{C}, x)\}$,

3. $N_{3}^{\mathbb{C}}(x)=\bigcap\{K \in \mathbb{C}: K \in \operatorname{MD}(\mathbb{C}, x)\}$,

4. $N_{4}^{\mathbb{C}}(x)=\bigcup\{K \in \mathbb{C}: K \in \operatorname{MD}(\mathbb{C}, x)\}=\bigcup\{K \in \mathbb{C}: K \in \mathscr{C}(\mathbb{C}, x)\}$.

All four neighborhood operators are reflexive. The operator $N_{4}^{\mathbb{C}}$ is symmetric and the operators $N_{1}^{\mathbb{C}}$ and $N_{3}^{\mathbb{C}}$ are transitive operators.

On the other hand, given a covering $\mathbb{C}$, Yao and Yao [28] also considered six coverings derived from $\mathbb{C}$ :

1. $\mathbb{C}_{1}=\bigcup\{\operatorname{md}(\mathbb{C}, x): x \in U\}$,

2. $\mathbb{C}_{2}=\bigcup\{\operatorname{MD}(\mathbb{C}, x): x \in U\}$,

3. $\mathbb{C}_{3}=\{\bigcap \operatorname{md}(\mathbb{C}, x): x \in U\}=\{\bigcap \mathscr{C}(\mathbb{C}, x): x \in U\}=\left\{N_{1}^{\mathbb{C}}: x \in U\right\}$,

4. $\mathbb{C}_{4}=\{\bigcup \operatorname{MD}(\mathbb{C}, x): x \in U\}=\{\bigcup \mathscr{C}(\mathbb{C}, x): x \in U\}=\left\{N_{4}^{\mathbb{C}}(x): x \in U\right\}$,

5. $\mathbb{C}_{\cap}=\mathbb{C} \backslash\left\{K \in \mathbb{C}:\left(\exists \mathbb{C}^{\prime} \subseteq \mathbb{C} \backslash\{K\}\right)\left(K=\bigcap \mathbb{C}^{\prime}\right)\right\}$,

6. $\mathbb{C}_{\cup}=\mathbb{C} \backslash\left\{K \in \mathbb{C}:\left(\exists \mathbb{C}^{\prime} \subseteq \mathbb{C} \backslash\{K\}\right)\left(K=\bigcup \mathbb{C}^{\prime}\right)\right\}$.

The idea behind the first two coverings is similar to the rationale for $N_{1}^{\mathbb{C}}, N_{2}^{\mathbb{C}}, N_{3}^{\mathbb{C}}$ and $N_{4}^{\mathbb{C}}$. By taking the union, the extreme neighborhood systems $\{\operatorname{md}(\mathbb{C}, x): x \in U\}$ and $\{\operatorname{MD}(\mathbb{C}, x): x \in U\}$ yield new coverings. Note that this is not the case when taking the intersection. Coverings $\mathbb{C}_{3}$ and $\mathbb{C}_{4}$ are directly related with neighborhood operators $N_{1}^{\mathbb{C}}$ and $N_{4}^{\mathbb{C}}$. Coverings $\mathbb{C}_{\cap}$ and $\mathbb{C}_{\cup}$ eliminate intersection reducible elements and union reducible elements from the covering, respectively. An intersection reducible element of a covering $\mathbb{C}$ is an element $K \in \mathbb{C}$ such that there exists $\mathbb{C}^{\prime} \subseteq \mathbb{C} \backslash\{K\}$ with $K=\bigcap \mathbb{C}^{\prime}$, while a union reducible element of $\mathbb{C}$ is an element $K \in \mathbb{C}$ such that there exists $\mathbb{C}^{\prime} \subseteq \mathbb{C} \backslash\{K\}$ with $K=\bigcup \mathbb{C}^{\prime}$

The equality $\mathbb{C}_{1}=\mathbb{C}_{\cup}$ was established in [20], while the other coverings are different in general. Also, note that $\mathbb{C}_{1}, \mathbb{C}_{2}$ and $\mathbb{C}_{\cap}$ are subcoverings of $\mathbb{C}$, while $\mathbb{C}_{3}$ and $\mathbb{C}_{4}$ are not. Furthermore, $\mathbb{C}_{2}$ is a subcovering of $\mathbb{C}_{\cap}$.

When combining the four neighborhood operators and the six coverings (one original and five derived ones), we obtain twenty-four combinations $N_{i}^{\mathbb{C}_{j}}$ for $N_{i} \in\left\{N_{1}, N_{2}, N_{3}, N_{4}\right\}$ and $\mathbb{C}_{j} \in\left\{\mathbb{C}, \mathbb{C}_{1}, \mathbb{C}_{2}, \mathbb{C}_{3}, \mathbb{C}_{4}, \mathbb{C}_{\cap}\right\}$. However, in [4], it is shown that there are only thirteen different groups of neighborhood operators. These groups are shown in Table 1.

Table 1: Neighborhood operators based on coverings

\begin{tabular}{ll|ll}
\hline Group & Operators & Group & Operators \\
\hline$A$. & $N_{1}^{\mathbb{C}}, N_{1}^{\mathbb{C}_{1}}, N_{1}^{\mathbb{C}_{3}}, N_{2}^{\mathbb{C}_{3}}, N_{1}^{\mathbb{C}_{n}}$ & $H$. & $N_{4}^{\mathbb{C}}, N_{2}^{\mathbb{C}_{2}}, N_{4}^{\mathbb{C}_{2}}, N_{4}^{\mathbb{C}_{n}}$ \\
\hline$B$. & $N_{3}^{\mathbb{C}_{1}}$ & $I$. & $N_{2}^{\mathbb{C}_{4}}$ \\
\hline$C$. & $N_{3}^{\mathbb{C}_{3}}$ & J. & $N_{3}^{\mathbb{C}_{4}}$ \\
\hline$D$. & $N_{4}^{\mathbb{C}_{3}}$ & $K$. & $N_{4}^{\mathbb{C}_{4}}$ \\
\hline$E$. & $N_{2}^{\mathbb{C}}, N_{2}^{\mathbb{C}_{1}}$ & $L$. & $N_{4}^{\mathbb{C}_{1}}$ \\
\hline$F$. & $N_{3}^{\mathbb{C}}, N_{1}^{\mathbb{C}_{2}}, N_{3}^{\mathbb{C}_{2}}, N_{3}^{\mathbb{C}_{n}}$ & $M$. & $N_{2}^{\mathbb{C}_{n}}$ \\
\hline$G$. & $N_{1}^{\mathbb{C}_{4}}$ & & \\
\hline
\end{tabular}

Besides equalities between neighborhood operators, [4] studied partial order relations between the operators, leading to the lattice given in Figure 1, with respect to the partial order relation $\leq$ defined as follows: let $N$ and $N^{\prime}$ be two neighborhood operators, then $N \leq N^{\prime}$ if and only if $\forall x, y \in U: x \in N(y) \Rightarrow x \in N^{\prime}(y)$. 
Figure 1: Lattice of the neighborhood operators from Table 1

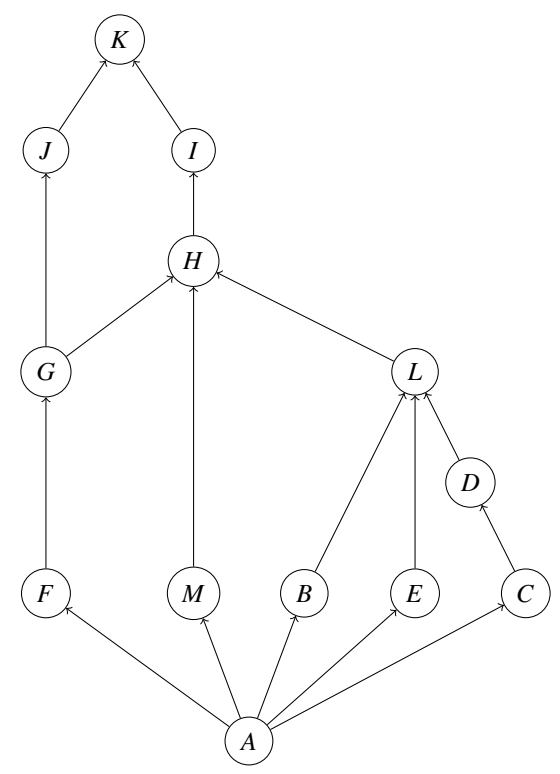

In the next section, we will define the fuzzy neigbhorhood system, the fuzzy minimal-description system and the fuzzy maximal-description system of an element $x \in U$ based on a fuzzy covering $\mathbb{C}$, which will lead us to fuzzy extensions of the four neighborhood operators and the six derived coverings. To end this section, we recall the definition of a fuzzy covering. Different definitions are proposed in [7, 13]. However, we will use the following one:

Definition 1. Let $\mathscr{F}(U)$ denote the collection of fuzzy subsets of $U$ and let I be an (infinite) index set. A collection $\mathbb{C}=\left\{K_{i} \in \mathscr{F}(U): K_{i} \neq \emptyset, i \in I\right\}$ is called a fuzzy covering, if for all $x \in U$ there exists a $K \in \mathbb{C}$ such that $K(x)=1$.

Note that for infinite coverings, this definition guarantees for any $x \in U$ the existence of a set $K \in \mathbb{C}$ to which $x$ fully belongs, which is not the case with the proposals of $[7,13]$.

\section{Fuzzy neighborhood operators based on a fuzzy covering}

In this section, we discuss the notion of fuzzy neighborhood operators in rough set theory and we propose definitions for the fuzzy extensions of the three neighborhood systems and for the four neighborhood operators previously considered. Moreover, we discuss which properties the different fuzzy neighborhood operators fulfil.

In the most general setting, a fuzzy neighborhood operator in the context of rough sets is defined as follows:

Definition 2. A fuzzy neighborhood operator is a mapping $N: U \rightarrow \mathscr{F}(U)$.

This means that a fuzzy neighborhood operator associates a fuzzy set $N(x)$ to every element $x \in U$. In equivalent terms, fuzzy neighborhood operators $N$ on $U$ are in one-to-one correspondence with fuzzy binary relations $R$ on $U$, just by taking $N(x)(y)=R(x, y)$ for all $x, y \in U$. Analogously to the crisp setting, we will assume in this paper that a fuzzy neighborhood operator is reflexive, i.e., $N(x)(x)=1$ for all $x \in U$, that is, an operator arising from a reflexive fuzzy relation. Sometimes we will be also considering fuzzy neighborhood operators satisfying additional properties, that in turn will clearly be in correspondence with analogous properties of the associated fuzzy binary relations. Namely, a fuzzy neighborhood operator is called symmetric if $N(x)(y)=N(y)(x)$ for all $x, y \in U$, i.e., the degree in which $y$ belongs to the neighborhood of $x$ equals the degree in which $x$ belongs to the neighborhood of $y$. Moreover, given a t-norm $\mathscr{T}$, we call a fuzzy neighborhood operator $N \mathscr{T}$-transitive if for all $x, y, z \in U$ holds that

$$
\mathscr{T}(N(x)(y), N(y)(z)) \leq N(x)(z) .
$$


If $N$ is $\mathscr{T}_{M}$-transitive, with $\mathscr{T}_{M}$ the minimum operator, then the membership degree of $z$ belonging to the neighborhood of $x$ will be at least equal to the minimum of the membership degree of $y$ to $N(x)$ and the membership degree of $z$ to $N(y)$, for each element $y \in U$.

\subsection{Fuzzy minimal and maximal descriptions}

In order to propose fuzzy extensions of the crisp neighborhood operators given a fuzzy covering $\mathbb{C}$, we first introduce the following extension of the neighborhood system of $x \in U$, which is the collection of all fuzzy sets in the fuzzy covering in which $x$ has a strict positive membership degree.

Definition 3. Let $\mathbb{C}$ be a fuzzy covering and $x \in U$, then the fuzzy neighborhood system of $x$ is given by

$$
\mathscr{C}(\mathbb{C}, x)=\{K \in \mathbb{C}: K(x)>0\} .
$$

By definition of a fuzzy covering, there is always a set $K \in \mathbb{C}$ with $K(x)=1$, hence, $\mathscr{C}(\mathbb{C}, x)$ is not empty. It is easy to see that if $\mathbb{C}$ is crisp, the neighborhood system $\mathscr{C}(\mathbb{C}, x)$ given in Equation (1) is obtained. The fuzzy minimal and maximal descriptions of $x$ are obtained as follows: in every non-zero membership degree that is reached by $x$ in $\mathbb{C}$, we take the minimal, resp. maximal, sets. This means that for all $\alpha \in\{K(x): K \in \mathbb{C}, K(x)>0\}$ there exist $K_{1} \in \operatorname{md}(\mathbb{C}, x)$ and $K_{2} \in \operatorname{MD}(\mathbb{C}, x)$ with $K_{1}(x)=K_{2}(x)=\alpha$.

Definition 4. Let $\mathbb{C}$ be a fuzzy covering and $x \in U$, then the fuzzy minimal description of $x$ is given by

$$
\operatorname{md}(\mathbb{C}, x)=\{K \in \mathscr{C}(\mathbb{C}, x):(\forall S \in \mathscr{C}(\mathbb{C}, x))(S(x)=K(x), S \subseteq K \Rightarrow S=K)\}
$$

and the fuzzy maximal description of $x$ is given by

$$
\operatorname{MD}(\mathbb{C}, x)=\{K \in \mathscr{C}(\mathbb{C}, x):(\forall S \in \mathscr{C}(\mathbb{C}, x))(S(x)=K(x), S \supseteq K \Rightarrow S=K)\} .
$$

If $\mathbb{C}$ is crisp, Equations (2) and (3) are obtained. Note that $\mathscr{C}(\mathbb{C}, x), \operatorname{md}(\mathbb{C}, x)$ and $\operatorname{MD}(\mathbb{C}, x)$ are all collections of fuzzy sets and that both $\operatorname{md}(\mathbb{C}, x)$ and $\operatorname{MD}(\mathbb{C}, x)$ are subsets of $\mathscr{C}(\mathbb{C}, x)$. We will illustrate the minimal and maximal descriptions in the following example.

Example 1. Let $U=\{x, y\}$ and $\mathbb{C}=\left\{K_{1}, K_{2}, K_{3}, K_{4}, K_{5}\right\}$ such that $K_{1}=1 / x+0.5 / y, K_{2}=0.7 / x+1 / y, K_{3}=$ $0.7 / x+0.5 / y, K_{4}=0.7 / x+0.2 / y$ and $K_{5}=0 / x+0.5 / y$. Then $\mathscr{C}(\mathbb{C}, x)=\left\{K_{1}, K_{2}, K_{3}, K_{4}\right\}, \operatorname{md}(\mathbb{C}, x)=\left\{K_{1}, K_{4}\right\}$ and $\operatorname{MD}(\mathbb{C}, x)=\left\{K_{1}, K_{2}\right\}$. On the other hand, $\mathscr{C}(\mathbb{C}, y)=\mathbb{C}$, $\operatorname{md}(\mathscr{C}, y)=\left\{K_{2}, K_{4}, K_{5}\right\}$ and $\operatorname{MD}(\mathbb{C}, y)=\left\{K_{1}, K_{2}, K_{4}\right\}$.

Due to the construction of the minimal and maximal descriptions, we keep the following property:

Proposition 2. Let $\mathbb{C}$ be a fuzzy covering such that any descending (resp. ascending) chain is closed under infimum (resp. supremum), i.e., for any set $\left\{K_{i} \in \mathbb{C}: i \in I\right\}$ with $K_{i+1} \subseteq K_{i}\left(\right.$ resp. $\left.K_{i+1} \supseteq K_{i}\right)$, then

$$
\inf _{i \in I} K_{i}=\bigcap_{i \in I} K_{i} \in \mathbb{C}\left(\text { resp. } \sup _{i \in I} K_{i}=\bigcup_{i \in I} K_{i} \in \mathbb{C}\right) \text {. }
$$

Let $K \in \mathscr{C}(\mathbb{C}, x)$, then there exist $K_{1} \in \operatorname{md}(\mathbb{C}, x)$ (resp. $K_{2} \in \operatorname{MD}(\mathbb{C}, x)$ ) such that $K_{1}(x)=K(x)$ and $K_{1} \subseteq K$ (resp. $K_{2}(x)=K(x)$ and $\left.K \subseteq K_{2}\right)$.

Proof. Since $K \in \mathscr{C}(\mathbb{C}, x), K(x)>0$. If $K \notin \operatorname{md}(\mathbb{C}, x)$, then by definition, there exists $K^{1} \in \mathbb{C}$ with $K^{1}(x)=K(x)$ and $K^{1} \subsetneq K$. If $K^{1} \notin \operatorname{md}(\mathbb{C}, x)$, then by definition, there exists $K^{2} \in \mathbb{C}$ with $K^{2}(x)=K^{1}(x)=K(x)$ and $K^{2} \subsetneq K^{1} \subsetneq K$. Continuing, as descending chains in $\mathbb{C}$ are closed under infimum, there exists $K_{1} \in \mathbb{C}$ such that $K_{1}=\inf _{i \in I} K^{i}$ with $K_{1}(x)=K(x)$ and $K_{1}(y) \leq K(y)$ for all $y \neq x$ and there is no set smaller in $\mathbb{C}$ than $K_{1}$ with these properties, therefore, $K_{1} \in \operatorname{md}(\mathbb{C}, x)$.

Similarly, we can find a $K_{2} \in \operatorname{MD}(\mathbb{C}, x)$ such that $K_{2}(x)=K(x)$ and $K \subseteq K_{2}$. 
Under the hypothesis of Proposition 2, there always exists a $K_{1} \in \operatorname{md}(\mathbb{C}, x)$ and $K_{2} \in \operatorname{MD}(\mathbb{C}, x)$ such that $K_{1}(x)=$ $K_{2}(x)=1$. Note that the condition of Proposition 2 holds whenever the fuzzy covering $\mathbb{C}$ is finite. As this proposition is a motivation for the fuzzy minimal and maximal descriptions, we will often explicitly assume that $\mathbb{C}$ is finite in order to apply this property. Although the condition of a finite $\mathbb{C}$ is stronger than the condition on $\mathbb{C}$ provided in Proposition 2 , we will often assume the former, as it is a more suitable condition for applications. Note that the condition on $\mathbb{C}$ in Proposition 2 is necessary, as illustrated in the next example:

Example 2. Let $U=\{x, y\}$ with $\mathbb{C}=\left\{K_{n}: n \in \mathbb{N} \backslash\{0\}\right\} \cup\left\{K^{*}=0.7 / x+0.1 / y\right\}$ such that $K_{n}(x)=1$ and $K_{n}(y)=\frac{1}{n}$. As $K^{*}$ is the only set in $\mathbb{C}$ with $K^{*}(x)=0.7, K^{*} \in \operatorname{md}(\mathbb{C}, x)$. However, as for all $n \in \mathbb{N} \backslash\{0\}$ it holds that $K_{n+1} \subseteq K_{n}$, $K_{n} \notin \operatorname{md}(\mathbb{C}, x)$. Therefore, there is no set $K$ in $\operatorname{md}(\mathbb{C}, x)$ with $K(x)=1$.

\subsection{Definitions of fuzzy neighborhood operators}

Given the definitions of the neighborhood system and the minimal and maximal description of $x \in U$ for a given fuzzy covering $\mathbb{C}$, we can now introduce fuzzy extensions of the four crisp neighborhood operators $N_{1}^{\mathbb{C}}, N_{2}^{\mathbb{C}}, N_{3}^{\mathbb{C}}$ and $N_{4}^{\mathbb{C}}$.

\subsubsection{Operator $N_{1}^{\mathbb{C}}$}

To introduce a fuzzy extension for the operator $N_{1}^{\mathbb{C}}$, we can rewrite the condition $y \in \bigcap \mathscr{C}(\mathbb{C}, x)$ as

$$
\forall K \in \mathbb{C}: x \in K \Rightarrow y \in K .
$$

A natural extension of this definition follows from replacing $\forall$ by the infimum operator, $x \in K$ by the membership degree $K(x)$ and $\Rightarrow$ by an implicator ${ }^{1} \mathscr{I}$.

Definition 5. Let $\mathbb{C}$ be a fuzzy covering and $\mathscr{I}$ an implicator, then $N_{1}^{\mathbb{C}}: U \rightarrow \mathscr{F}(U): x \mapsto N_{1}^{\mathbb{C}}(x)$ is a fuzzy neighborhood operator, for which the fuzzy neighborhood $N_{1}^{\mathbb{C}}(x)$ is defined by

$$
N_{1}^{\mathbb{C}}(x): U \rightarrow[0,1]: y \mapsto \inf _{K \in \mathbb{C}} \mathscr{I}(K(x), K(y)) .
$$

If the covering $\mathbb{C}$ is a crisp covering, then the fuzzy neighborhood of $x$ described in Equation (7) coincides with the crisp neighborhood of $x$. Indeed, for $x, y \in U$, the membership degree $N_{1}^{\mathbb{C}}(x)(y)$ is either 0 or 1 . Moreover, it holds that

$$
\begin{aligned}
N_{1}^{\mathbb{C}}(x)(y)=1 & \Leftrightarrow \inf _{K \in \mathbb{C}} \mathscr{I}(K(x), K(y))=1 \\
& \Leftrightarrow \quad \forall K \in \mathbb{C}: \mathscr{I}(K(x), K(y))=1 \\
& \Leftrightarrow \quad \forall K \in \mathbb{C}: K(x)=1 \Rightarrow K(y)=1 \\
& \Leftrightarrow \quad \forall K \in \mathbb{C}: x \in K \Rightarrow y \in K \\
& \Leftrightarrow y \in \bigcap \mathscr{C}(\mathbb{C}, x)
\end{aligned}
$$

To construct the operator $N_{1}^{\mathbb{C}}$, we have used the characterization $y \in \bigcap \mathscr{C}(\mathbb{C}, x)$ of the crisp neighborhood $N_{1}^{\mathbb{C}}(x)$. Next, we prove that the characterization $y \in \bigcap \operatorname{md}(\mathbb{C}, x)$ yields the same fuzzy neighborhood operator.

Proposition 3. Let $\mathbb{C}$ be a finite fuzzy covering and $\mathscr{I}$ be an implicator, then for all $x, y \in U$ it holds that

$$
\inf _{K \in \mathbb{C}} \mathscr{I}(K(x), K(y))=\inf _{K \in \mathscr{C}(\mathbb{C}, x)} \mathscr{I}(K(x), K(y))=\inf _{K \in \operatorname{md}(\mathbb{C}, x)} \mathscr{I}(K(x), K(y))
$$

\footnotetext{
${ }^{1}$ An implicator is a mapping $\mathscr{I}:[0,1]^{2} \rightarrow[0,1]$ which is decreasing in the first and increasing in the second argument and satisfies $\mathscr{I}(0,0)=$ $\mathscr{I}(1,1)=1$ and $\mathscr{I}(1,0)=0[1]$
} 
Proof. First note that if $K(x)=0$, then $\mathscr{I}(K(x), K(y))=1$, hence

$$
\inf _{K \in \mathbb{C}} \mathscr{I}(K(x), K(y))=\inf _{K \in \mathscr{C}(\mathbb{C}, x)} \mathscr{I}(K(x), K(y)) .
$$

Since $\operatorname{md}(\mathbb{C}, x) \subseteq \mathscr{C}(\mathbb{C}, x)$, we have that

$$
\inf _{K \in \mathbb{C}} \mathscr{I}(K(x), K(y))=\min \left(\inf _{K \in \operatorname{md}(\mathbb{C}, x)} \mathscr{I}(K(x), K(y)), \inf _{K \in \mathscr{C}(\mathbb{C}, x) \backslash \operatorname{md}(\mathbb{C}, x)} \mathscr{I}(K(x), K(y))\right) .
$$

If $K \in \mathscr{C}(\mathbb{C}, x) \backslash \operatorname{md}(\mathbb{C}, x)$, then there exists a $K^{\prime} \in \operatorname{md}(\mathbb{C}, x)$ such that $K^{\prime} \subseteq K$ and $K^{\prime}(x)=K(x)$. Therefore, for all $y \in U$,

$$
\mathscr{I}(K(x), K(y))=\mathscr{I}\left(K^{\prime}(x), K(y)\right) \geq \mathscr{I}\left(K^{\prime}(x), K^{\prime}(y)\right) .
$$

Hence, we can conclude that $\inf _{K \in \operatorname{md}(\mathbb{C}, x)} \mathscr{I}(K(x), K(y)) \leq \inf _{K \in \mathscr{C}(\mathbb{C}, x) \backslash \operatorname{md}(\mathbb{C}, x)} \mathscr{I}(K(x), K(y))$ and thus,

$$
\inf _{K \in \mathbb{C}} \mathscr{I}(K(x), K(y))=\inf _{K \in \operatorname{md}(\mathbb{C}, x)} \mathscr{I}(K(x), K(y)) .
$$

Note that we assume the fuzzy covering $\mathbb{C}$ to be finite, in order to be able to apply Proposition 2.

\subsubsection{Operator $N_{2}^{\mathbb{C}}$}

For the fuzzy extension of $N_{2}^{\mathbb{C}}$, note that the condition $y \in \bigcup \operatorname{md}(\mathbb{C}, x)$ can be rewritten as

$$
\exists K \in \operatorname{md}(\mathbb{C}, x): x \in K \wedge y \in K .
$$

A natural extension of this definition follows from replacing $\exists$ by the supremum operator, $x \in K$ by the membership degree $K(x)$ and $\wedge$ by a t-norm $\mathscr{T}$.

Definition 6. Let $\mathbb{C}$ be a fuzzy covering and $\mathscr{T}$ a t-norm, then $N_{2}^{\mathbb{C}}: U \rightarrow \mathscr{F}(U): x \mapsto N_{2}^{\mathbb{C}}(x)$ is a fuzzy neighborhood operator, for which the fuzzy neighborhood $N_{2}^{\mathbb{C}}(x)$ is defined by

$$
N_{2}^{\mathbb{C}}(x): U \rightarrow[0,1]: y \mapsto \sup _{K \in \operatorname{md}(\mathbb{C}, x)} \mathscr{T}(K(x), K(y)) .
$$

Note that if the covering $\mathbb{C}$ is a crisp covering, the fuzzy minimal description of $x$ coincides with the crisp minimal description of $x$. Moreover, in that case, the fuzzy neighborhood defined in Equation (8) coincides with the crisp neighborhood $N_{2}^{\mathbb{C}}(x)$.

\subsubsection{Operator $N_{3}^{\mathbb{C}}$}

In the crisp case, the condition $y \in \bigcap \operatorname{MD}(\mathbb{C}, x)$ can be rewritten as

$$
\forall K \in \operatorname{MD}(\mathbb{C}, x): x \in K \Rightarrow y \in K .
$$

As with the operator $N_{1}^{\mathbb{C}}$, a natural extension of this definition follows from replacing $\forall$ by the infimum operator, $x \in K$ by the membership degree $K(x)$ and $\Rightarrow$ by an implicator $\mathscr{I}$.

Definition 7. Let $\mathbb{C}$ be a fuzzy covering and $\mathscr{I}$ an implicator, then $N_{3}^{\mathbb{C}}: U \rightarrow \mathscr{F}(U): x \mapsto N_{3}^{\mathbb{C}}(x)$ is a fuzzy neighborhood operator, for which the fuzzy neighborhood $N_{3}^{\mathbb{C}}(x)$ is defined by

$$
N_{3}^{\mathbb{C}}(x): U \rightarrow[0,1]: y \mapsto \inf _{K \in \mathrm{MD}(\mathbb{C}, x)} \mathscr{I}(K(x), K(y)) .
$$

Given a crisp covering $\mathbb{C}$, the fuzzy maximal description of $x$ coincides with the crisp maximal description of $x$ and the fuzzy neighborhood of $x$ described in Equation (9) coincides with the crisp neighborhood $N_{3}^{\mathbb{C}}(x)$. 


\subsubsection{Operator $N_{4}^{\mathbb{C}}$}

We can rewrite the characterization $y \in \bigcup \mathscr{C}(\mathbb{C}, x)$ as

$$
\exists K \in \mathbb{C}: x \in K \wedge y \in K .
$$

As with the operator $N_{2}^{\mathbb{C}}$, a natural extension of this definition follows from replacing $\exists$ by the supremum operator, $x \in K$ by the membership degree $K(x)$ and $\wedge$ by a t-norm $\mathscr{T}$.

Definition 8. Let $\mathbb{C}$ be a fuzzy covering and $\mathscr{T}$ a t-norm, then $N_{4}^{\mathbb{C}}: U \rightarrow \mathscr{F}(U): x \mapsto N_{4}^{\mathbb{C}}(x)$ is a fuzzy neighborhood operator, for which the fuzzy neighborhood $N_{4}^{\mathbb{C}}(x)$ is defined by

$$
N_{4}^{\mathbb{C}}(x): U \rightarrow[0,1]: y \mapsto \sup _{K \in \mathbb{C}} \mathscr{T}(K(x), K(y)) .
$$

It is easy to see that the fuzzy neighborhood of $x$ defined in Equation (10) is a fuzzy extension of the crisp neighborhood $N_{4}^{\mathbb{C}}(x)$.

Finally, we can prove the analogy of the crisp equality $\bigcup \mathscr{C}(\mathbb{C}, x)=\bigcup M D(\mathbb{C}, x)$ in the fuzzy setting.

Proposition 4. Let $\mathbb{C}$ be a finite fuzzy covering and $\mathscr{T}$ be a t-norm, then for all $x, y \in U$ it holds that

$$
\sup _{K \in \mathbb{C}} \mathscr{T}(K(x), K(y))=\sup _{K \in \mathscr{C}(\mathbb{C}, x)} \mathscr{T}(K(x), K(y))=\sup _{K \in \operatorname{MD}(\mathbb{C}, x)} \mathscr{T}(K(x), K(y)) .
$$

Proof. Analogously as in the proof of Proposition 3, we can prove that $\sup _{K \in \mathbb{C}} \mathscr{T}(K(x), K(y))=\sup _{K \in \mathscr{C}(\mathbb{C}, x)} \mathscr{T}(K(x), K(y))$ and $\sup _{K \in \mathrm{MD}(\mathbb{C}, x)} \mathscr{T}(K(x), K(y)) \geq \sup _{K \in \mathscr{C}(\mathbb{C}, x) \backslash \mathrm{MD}(\mathbb{C}, x)} \mathscr{T}(K(x), K(y))$. Hence, the supremum will be reached in $\mathrm{MD}(\mathbb{C}, x)$.

In the next section, we study the properties of the different fuzzy neighborhood operators.

\subsection{Properties of the fuzzy neighborhood operators}

In the crisp case, the neighborhood operators $N_{1}^{\mathbb{C}}$ and $N_{3}^{\mathbb{C}}$ are reflexive and transitive, while $N_{4}^{\mathbb{C}}$ is reflexive and symmetric and $N_{2}^{\mathbb{C}}$ is reflexive. The fuzzy neighborhood operators maintain these properties under some conditions on the fuzzy covering, the implicator and the t-norm.

First, we consider the reflexivity property.

Proposition 5. Let $\mathbb{C}$ be a fuzzy covering, $\mathscr{T}$ a $t$-norm and $\mathscr{I}$ an implicator which satisfies $\mathscr{I}(a, b)=1$ if $a \leq b$ for $a, b \in[0,1]$ (weak confinement principle). Then the operators $N_{1}^{\mathbb{C}}$ and $N_{3}^{\mathbb{C}}$ defined with $\mathscr{I}$ and the operator $N_{4}^{\mathbb{C}}$ defined with $\mathscr{T}$ are reflexive fuzzy neighborhood operators. The fuzzy neighborhood operator $\mathrm{N}_{2}^{\mathbb{C}}$ defined with $\mathscr{T}$ is reflexive if $\mathbb{C}$ is finite.

Proof. As $\mathscr{I}(a, a)=1$ for all $a \in[0,1]$, the operators $N_{1}^{\mathbb{C}}$ and $N_{3}^{\mathbb{C}}$ are reflexive. Moreover, let $x \in U$, then there exists $K \in \mathbb{C}$ such that $K(x)=1$. Hence, $N_{4}^{\mathbb{C}}(x)(x) \geq \mathscr{T}(K(x), K(x))=1$.

Now assume $\mathbb{C}$ is finite, then there exists $K_{1} \in \operatorname{md}(\mathbb{C}, x)$ with $K_{1}(x)=K(x)=1$ and $K_{1} \subseteq K$. Hence, $N_{2}^{\mathbb{C}}(x)(x) \geq$ $\mathscr{T}\left(K_{1}(x), K_{1}(x)\right)=1$, and thus, $N_{2}^{\mathbb{C}}$ is a reflexive operator.

Note that R-implicators satisfy the required condition on $\mathscr{I}$. Recall that the R-implicator $\mathscr{I}$ based on the $\mathrm{t}$ norm $\mathscr{T}$ is defined by $\forall a, b \in[0,1]: \mathscr{I}(a, b)=\sup \{c \in[0,1]: \mathscr{T}(a, c) \leq b\}$.

Second, the operators $N_{1}^{\mathbb{C}}$ and $N_{3}^{\mathbb{C}}$ are $\mathscr{T}$-transitive for a left-continuous t-norm $\mathscr{T}$ if the used implicator is the R-implicator of $\mathscr{T}$. In order to prove transitivity, we first consider the following well-known result: 
Lemma 1. [19] Let $\mathscr{T}$ be a left-continuous t-norm and $\mathscr{I}$ its $R$-implicator, then $\mathscr{T}(\mathscr{I}(a, b), \mathscr{I}(b, c)) \leq \mathscr{I}(a, c)$ for all $a, b, c \in[0,1]$.

Based on this lemma, we can prove the transitivity of the operators $N_{1}^{\mathbb{C}}$ and $N_{3}^{\mathbb{C}}$.

Proposition 6. Let $\mathbb{C}$ be a fuzzy covering and let $\mathscr{T}$ be a left-continuous $t$-norm and $\mathscr{I}$ its $R$-implicator used in the definition of $N_{1}^{\mathbb{C}}$ and $N_{3}^{\mathbb{C}}$, then for each $x, y, z \in U$ it holds that

$$
\begin{aligned}
& \mathscr{T}\left(N_{1}^{\mathbb{C}}(y)(x), N_{1}^{\mathbb{C}}(x)(z)\right) \leq N_{1}^{\mathbb{C}}(y)(z), \\
& \mathscr{T}\left(N_{3}^{\mathbb{C}}(y)(x), N_{3}^{\mathbb{C}}(x)(z)\right) \leq N_{3}^{\mathbb{C}}(y)(z),
\end{aligned}
$$

i.e., $N_{1}^{\mathbb{C}}$ and $N_{3}^{\mathbb{C}}$ are $\mathscr{T}$-transitive fuzzy neighborhood operators.

Proof. We have for each $x, y, z \in U$ that

$$
\begin{aligned}
\mathscr{T}\left(\inf _{K \in \mathbb{C}} \mathscr{I}(K(y), K(x)), \inf _{K \in \mathbb{C}} \mathscr{I}(K(x), K(z))\right) & \leq \inf _{K \in \mathbb{C}} \mathscr{T}(\mathscr{I}(K(y), K(x)), \mathscr{I}(K(x), K(z))) \\
& \leq \inf _{K \in \mathbb{C}} \mathscr{I}(K(y), K(z)),
\end{aligned}
$$

where in the last inequality Lemma 1 is used. The proof for $N_{3}^{\mathbb{C}}$ is similar.

Note that we have very explicitly used properties specific to a left-continuous t-norm and its R-implicator. We illustrate this with an example.

Example 3. Let $U=\{x, y, z\}$ and $\mathbb{C}=\left\{K_{1}, K_{2}\right\}$ with $K_{1}=1 / x+0.1 / y+1 / z$ and $K_{2}=0.5 / x+1 / y+0.2 / z$. Let $\mathscr{T}$ be the minimum operator $\mathscr{T}_{M}$ and $\mathscr{I}$ the Kleene-Dienes implicator defined by $\mathscr{I}(a, b)=\max (1-a, b)$ for $a, b \in[0,1]$, which is used to define $N_{1}^{\mathbb{C}}$ and $N_{3}^{\mathbb{C}}$. Then we have that

$$
\begin{aligned}
& N_{1}^{\mathbb{C}}(y)(x)=N_{3}^{\mathbb{C}}(y)(x)=0.5, \\
& N_{1}^{\mathbb{C}}(x)(z)=N_{3}^{\mathbb{C}}(x)(z)=0.5, \\
& N_{1}^{\mathbb{C}}(y)(z)=N_{3}^{\mathbb{C}}(y)(z)=0.2 .
\end{aligned}
$$

Therefore, we obtain that $\mathscr{T}(0.5,0.5)>0.2$. Hence, $N_{1}^{\mathbb{C}}$ and $N_{3}^{\mathbb{C}}$ defined with the Kleene-Dienes implicator are not $\mathscr{T}_{M}$-transitive.

Finally, we prove that the fuzzy neighborhood operator $N_{4}^{\mathbb{C}}$ is symmetric.

Proposition 7. Let $\mathbb{C}$ be a fuzzy covering and $N_{4}^{\mathbb{C}}$ based on $\mathscr{T}$, then for all $x, y \in U$ it holds that $N_{4}^{\mathbb{C}}(x)(y)=N_{4}^{\mathbb{C}}(y)(x)$. Proof. This follows immediately from the fact that a t-norm is commutative.

Although $N_{2}^{\mathbb{C}}$ is defined with a t-norm, the operator is not symmetric, as the minimal descriptions of $x$ and $y$ are not necessarily equal.

Next, we discuss different derived coverings of a fuzzy covering.

\section{Fuzzy coverings derived from a fuzzy covering}

Given a fuzzy covering $\mathbb{C}$, we introduce fuzzy extensions of the derived coverings $\mathbb{C}_{1}, \mathbb{C}_{2}, \mathbb{C}_{3}, \mathbb{C}_{4}, \mathbb{C}_{\cap}$ and $\mathbb{C}_{\cup}$.

Definition 9. Let $\mathbb{C}$ be a fuzzy covering, $\mathscr{T}$ a t-norm to construct operator $N_{4}^{\mathbb{C}}$ and $\mathscr{I}$ an implicator to construct operator $N_{1}^{\mathbb{C}}$, then define the following collections of fuzzy sets:

- $\mathbb{C}_{1}=\bigcup\{\operatorname{md}(\mathbb{C}, x): x \in U\}$, 
- $\mathbb{C}_{2}=\bigcup\{\mathrm{MD}(\mathbb{C}, x): x \in U\}$,

- $\mathbb{C}_{3}=\left\{N_{1}^{\mathbb{C}}(x): x \in U\right\}$,

- $\mathbb{C}_{4}=\left\{N_{4}^{\mathbb{C}}(x): x \in U\right\}$,

- $\mathbb{C}_{\cap}=\mathbb{C} \backslash\left\{K \in \mathbb{C}:\left(\exists \mathbb{C}^{\prime} \subseteq \mathbb{C} \backslash\{K\}\right)\left(K=\cap \mathbb{C}^{\prime}\right)\right\}$,

- $\mathbb{C}_{\cup}=\mathbb{C} \backslash\left\{K \in \mathbb{C}:\left(\exists \mathbb{C}^{\prime} \subseteq \mathbb{C} \backslash\{K\}\right)\left(K=\bigcup \mathbb{C}^{\prime}\right)\right\}$.

We illustrate these definitions in the following example.

Example 4. Let $U=\{x, y, z\}$ and $\mathbb{C}=\left\{K_{1}, K_{2}, K_{3}, K_{4}, K_{5}, K_{6}, K_{7}, K_{8}\right\}$ with $K_{1}=0.9 / x+0.9 / y+0.7 / z, K_{2}=0.9 / x+$ $1 / y+0.9 / z, K_{3}=0.9 / x+0.1 / y+0.8 / z, K_{4}=1 / x+0.9 / y+1 / z, K_{5}=1 / x+0 / y+0.6 / z, K_{6}=1 / x+1 / y+0.7 / z$, $K_{7}=1 / x+0 / y+0.5 / z$ and $K_{8}=1 / x+0.9 / y+0.7 / z$, then $\mathbb{C}_{1}=\mathbb{C}_{\cup}=\left\{K_{1}, K_{2}, K_{3}, K_{4}, K_{5}, K_{6}, K_{7}\right\}$ and $\mathbb{C}_{2}=\mathbb{C}_{\cap}=$ $\left\{K_{2}, K_{3}, K_{4}, K_{5}, K_{6}, K_{7}\right\}$. Moreover, if $\mathscr{T}$ is the minimum operator and $\mathscr{I}$ its $R$-implicator, then $\mathbb{C}_{3}=\{1 / x+0 / y+$ $0.5 / z, 0.9 / x+1 / y+0.7 / z, 1 / x+0 / y+1 / z\}$ and $\mathbb{C}_{4}=\{1 / x+1 / y+1 / z, 1 / x+1 / y+0.9 / z, 1 / x+0.9 / y+1 / z\}$.

Next, we will prove that the collections defined above are all fuzzy coverings if the original covering $\mathbb{C}$ is finite. First, we show that the collections $\mathbb{C}_{1}, \mathbb{C}_{2}$ and $\mathbb{C}_{\cup}$ are finite fuzzy subcoverings of $\mathbb{C}$.

Proposition 8. Let $\mathbb{C}$ be a finite fuzzy covering, then $\mathbb{C}_{1}, \mathbb{C}_{2}$ and $\mathbb{C} \cup$ are all finite fuzzy subcoverings of $\mathbb{C}$.

Proof. It is easy to see that all three collections are subsets of the covering $\mathbb{C}$, and that they are finite collections of non-empty fuzzy sets of $U$. We need to study whether the condition $\forall x \in U, \exists K_{j} \in \mathbb{C}_{j}: K_{j}(x)=1$ holds.

Take $x \in U$, then there exists $K \in \mathbb{C}$ such that $K(x)=1$. Hence, there exist $K_{1} \in \operatorname{md}(\mathbb{C}, x)$ and $K_{2} \in \operatorname{MD}(\mathbb{C}, x)$ such that $K_{1}(x)=K(x)=K_{2}(x)=1$ and $K_{1} \subseteq K \subseteq K_{2}$. Since $K_{1} \in \mathbb{C}_{1}$ and $K_{2} \in \mathbb{C}_{2}, \mathbb{C}_{1}$ and $\mathbb{C}_{2}$ are fuzzy coverings.

As for $\mathbb{C}_{\cup}$, assume $K \notin \mathbb{C} \cup$, then there exists a collection $\mathbb{C}^{\prime} \subseteq \mathbb{C} \backslash\{K\}$ such that $K=\cup \mathbb{C}^{\prime}$. Since $\mathbb{C}$ is finite, there exists a $K^{\prime} \in \mathbb{C}^{\prime}$ such that $K^{\prime}(x)=1$. Since we can choose the collection $\mathbb{C}^{\prime}$ in $\mathbb{C}_{\cup}$, there exists $K^{\prime} \in \mathbb{C}_{\cup}$ such that $K^{\prime}(x)=1$. Hence, $\mathbb{C}_{\cup}$ is a fuzzy covering.

The condition of finiteness for $\mathbb{C}$ is necessary, as for the coverings $\mathbb{C}_{1}$ and $\mathbb{C}_{2}$ Proposition 2 is used. The necessity of the condition for $\mathbb{C}_{\cup}$ is illustrated in the next example:

Example 5. Let $U=\{x\}$ and $\mathbb{C}=\left\{K_{n}: n \in \mathbb{N} \backslash\{0\}\right\} \cup\left\{K^{*}\right\}$ with $K_{n}(x)=1-\frac{1}{n}$ and $K^{*}(x)=1$. It holds that $\sup \left\{K_{n}(x): \mathbb{N} \backslash\{0\}\right\}=K^{*}(x)$, and thus, $K^{*} \notin \mathbb{C}_{\cup}$. Therefore, $\mathbb{C}_{\cup}$ is no fuzzy covering.

Next, we show that $\mathbb{C}_{\cap}$ is a fuzzy covering for an infinite covering $\mathbb{C}$.

Proposition 9. Let $\mathbb{C}$ be a fuzzy covering, then $\mathbb{C}_{\cap}$ is a fuzzy subcovering of $\mathbb{C}$.

Proof. By definition, $\mathbb{C}_{\cap}$ is a subset of $\mathbb{C}$ and therefore, it does not contain the empty set. Moreover, let $x \in U$ and $K \in \mathbb{C}$ with $K(x)=1$. Assume that $K \notin \mathbb{C}_{\cap}$, then there exists a collection $\mathbb{C}^{\prime} \subseteq \mathbb{C} \backslash\{K\}$ such that $K=\cap \mathbb{C}^{\prime}$. Since $K(x)=1$, it holds for all $K^{\prime} \in \mathbb{C}^{\prime}$ that $K^{\prime}(x)=1$. Since we can choose the collection $\mathbb{C}^{\prime}$ in $\mathbb{C}_{\cap}$, there exists $K^{\prime} \in \mathbb{C}_{\cap}$ such that $K^{\prime}(x)=1$. Hence, $\mathbb{C}_{\cap}$ is a fuzzy covering.

Furthermore, we prove that $\mathbb{C}_{3}$ and $\mathbb{C}_{4}$ are fuzzy coverings.

Proposition 10. Let $\mathbb{C}$ be a fuzzy covering, $\mathscr{T}$ a t-norm to construct $\mathbb{C}_{4}$ and $\mathscr{I}$ an implicator which satisfies the weak confinement principle to construct $\mathbb{C}_{3}$, then $\mathbb{C}_{3}$ and $\mathbb{C}_{4}$ are fuzzy coverings.

Proof. This follows immediately from the fact that $N_{1}^{\mathbb{C}}$ and $N_{4}^{\mathbb{C}}$ are reflexive.

As opposed to the fuzzy coverings $\mathbb{C}_{1}, \mathbb{C}_{2}, \mathbb{C}_{\cap}$ and $\mathbb{C}_{\cup}$ which are subcoverings of $\mathbb{C}$, it is possible that the fuzzy coverings $\mathbb{C}_{3}$ and $\mathbb{C}_{4}$ have no overlap with the original covering $\mathbb{C}$. Moreover, note that the cardinality of $\mathbb{C}_{3}$ and $\mathbb{C}_{4}$ is at most the cardinality of $U$, while the cardinality of the other four derived coverings will be at most the cardinality of $\mathbb{C}$.

Additionally, we prove that $\mathbb{C}_{2} \subseteq \mathbb{C}_{\cap}$ and $\mathbb{C}_{\cup}=\mathbb{C}_{1}$ if $\mathbb{C}$ is finite. 
Proposition 11. Let $\mathbb{C}$ be a finite fuzzy covering, then $\mathbb{C}_{2}$ is a fuzzy subcovering of $\mathbb{C}_{\cap}$.

Proof. Let $K \in \mathbb{C}_{2}$, then there exists $x \in U$ such that $K \in \operatorname{MD}(\mathbb{C}, x)$. If $K \notin \mathbb{C}_{\cap}$, then there exists a collection $\mathbb{C}^{\prime} \subseteq$ $\mathbb{C} \backslash\{K\}$ such that $K=\bigcap \mathbb{C}^{\prime}$. We can choose the collection $\mathbb{C}^{\prime}$ in $\mathbb{C}_{\cap}$. Since $\mathbb{C}$ is finite, take $K^{\prime} \in \mathbb{C}^{\prime}$ such that $K^{\prime}(x)=K(x)>0$. Because $K \subseteq K^{\prime}, K(x)=K^{\prime}(x)$ and $K \in \operatorname{MD}(\mathbb{C}, x)$, we have that $K=K^{\prime}$. Hence, $K \in \mathbb{C}_{\cap}$.

Note that $\mathbb{C}_{2}$ is not necessarily a subset of $\mathbb{C}_{\cap}$ if $\mathbb{C}$ is infinite:

Example 6. Let $U=\{x\}$ and the covering $\mathbb{C}=\left\{K_{n}: n \in \mathbb{N} \backslash\{0,1\}\right\} \cup\left\{K^{*}\right\}$ is defined by $K_{n}(x)=\frac{1}{2}+\frac{1}{n}$ and $K^{*}(x)=\frac{1}{2}$, then $\inf \left\{K_{n}(x): n \in \mathbb{N} \backslash\{0,1\}\right\}=K^{*}(x)$, thus $K^{*} \notin \mathbb{C}_{\cap}$. Since the membership degree of $x$ in every fuzzy set of $\mathbb{C} i s$ different, we have that $\mathbb{C}_{2}=\mathbb{C}$ and therefore, $\mathbb{C}_{2}$ is not a subset of $\mathbb{C}_{\cap}$.

Proposition 12. Let $\mathbb{C}$ be a finite fuzzy covering, then $\mathbb{C}_{\cup}=\mathbb{C}_{1}$.

Proof. First, let $K \in \mathbb{C}_{1}$, then there exists $x \in U$ such that $K \in \operatorname{md}(\mathbb{C}, x)$. If $K \notin \mathbb{C} \cup$, then there exists a collection $\mathbb{C}^{\prime} \subseteq \mathbb{C} \backslash\{K\}$ such that $K=\cup \mathbb{C}^{\prime}$. We can choose the collection $\mathbb{C}^{\prime}$ in $\mathbb{C}$. Since $\mathbb{C}$ is finite, take $K^{\prime} \in \mathbb{C}^{\prime}$ such that $K^{\prime}(x)=K(x)>0$. Because $K^{\prime} \subseteq K, K^{\prime}(x)=K(x)$ and $K \in \operatorname{md}(\mathbb{C}, x)$, we have that $K=K^{\prime}$. Hence, $K \in \mathbb{C}_{\cup}$.

Second, let $K \in \mathbb{C}_{\cup}$ and assume that $K \notin \mathbb{C}_{1}$, then for all $x \in U, K \notin \operatorname{md}(\mathbb{C}, x)$. Since $K$ is not empty, there exists $x \in U$ such that $K(x)>0$. Hence, there exists $K_{x} \in \operatorname{md}(\mathbb{C}, x)$ with $K_{x}(x)=K(x)$ and $K_{x} \subsetneq K$. Therefore,

$$
\bigcup_{x \in U: K(x)>0} K_{x} \subseteq K .
$$

On the other hand, for each $z \in U$ we have that

$$
\sup _{x \in U: K(x)>0} K_{x}(z) \geq K(z)
$$

because if $K(z)>0$, then $\sup _{x \in U: K(x)>0} K_{x}(z) \geq K_{z}(z)=K(z)$ and if $K(z)=0$, it holds trivially. Hence, we conclude that

$$
K=\bigcup_{x \in U: K(x)>0} K_{x}
$$

where $\left\{K_{x}: x \in U, K(x)>0\right\} \subseteq \mathbb{C} \backslash\{K\}$, which means that $K \notin \mathbb{C}_{\cup}$. This is a contradiction, thus, $K \in \mathbb{C}_{1}$.

Note that the finiteness condition is necessary, as otherwise $\mathbb{C}_{1}$ and $\mathbb{C}_{\cup}$ are not coverings.

We can conclude that a finite fuzzy covering $\mathbb{C}$ yields five derived fuzzy coverings $\mathbb{C}_{1}=\mathbb{C}_{\cup}, \mathbb{C}_{2}, \mathbb{C}_{3}, \mathbb{C}_{4}$ and $\mathbb{C}_{\cap}$. These six fuzzy coverings (one original and five derived ones), together with the four fuzzy neighborhood operators, result in twenty-four combinations of fuzzy neighborhood operators based on a finite fuzzy covering. In the next section, we discuss whether the equalities stated in Table 1 are still maintained.

\section{Equalities between fuzzy neighborhood operators based on a finite fuzzy covering}

In this section, we discuss equalities between fuzzy neighborhood operators based on a covering $\mathbb{C}$. We will assume that this covering $\mathbb{C}$ is finite, therefore, the fuzzy covering $\mathbb{C}_{\cup}$ is disregarded since it is equal to the fuzzy covering $\mathbb{C}_{1}$. First note that when two neighborhood operators are different in the crisp case, they are also different in the fuzzy setting. Therefore, we only need to study whether the equalities of groups $A, E, F$ and $H$ of Table 1 are maintained.

We start with the following observations.

Proposition 13. Let $\mathbb{C}$ be a finite fuzzy covering, then for all $x \in U$ it holds that 
1. $\operatorname{md}\left(\mathbb{C}_{1}, x\right)=\operatorname{md}(\mathbb{C}, x)$,

2. $\operatorname{MD}\left(\mathbb{C}_{2}, x\right)=\operatorname{MD}(\mathbb{C}, x)$,

3. $\operatorname{MD}\left(\mathbb{C}_{\cap}, x\right)=\operatorname{MD}(\mathbb{C}, x)$.

Proof. 1. Take $x \in U$. If $K \in \operatorname{md}(\mathbb{C}, x)$, then $K \in \mathbb{C}_{1}$ and $K(x)>0$. Let $K^{\prime} \in \mathbb{C}_{1}$ with $K^{\prime}(x)=K(x)>0$ and $K^{\prime} \subseteq K$. Since $K^{\prime} \in \mathbb{C}$ and $K \in \operatorname{md}(\mathbb{C}, x)$, it holds that $K=K^{\prime}$. Hence, $K \in \operatorname{md}\left(\mathbb{C}_{1}, x\right)$.

On the other hand, if $K \in \operatorname{md}\left(\mathbb{C}_{1}, x\right)$, then $K \in \mathbb{C}_{1} \subseteq \mathbb{C}$ and $K(x)>0$. Hence, there exists $K^{\prime} \in \operatorname{md}(\mathbb{C}, x)$ with $K^{\prime}(x)=K(x)$ and $K^{\prime} \subseteq K$. Since $K^{\prime} \in \mathbb{C}_{1}$ and $K \in \operatorname{md}\left(\mathbb{C}_{1}, x\right)$, it holds that $K=K^{\prime}$. Hence, $K \in \operatorname{md}(\mathbb{C}, x)$.

2. Take $x \in U$. If $K \in \operatorname{MD}(\mathbb{C}, x)$, then $K \in \mathbb{C}_{2}$ and $K(x)>0$. Let $K^{\prime} \in \mathbb{C}_{2}$ with $K^{\prime}(x)=K(x)>0$ and $K \subseteq K^{\prime}$. Since $K^{\prime} \in \mathbb{C}$ and $K \in \operatorname{MD}(\mathbb{C}, x)$, it holds that $K=K^{\prime}$. Hence, $K \in \operatorname{MD}\left(\mathbb{C}_{2}, x\right)$.

On the other hand, if $K \in \operatorname{MD}\left(\mathbb{C}_{2}, x\right)$, then $K \in \mathbb{C}_{2} \subseteq \mathbb{C}$ and $K(x)>0$. Hence, there exists $K^{\prime} \in \operatorname{MD}(\mathbb{C}, x)$ with $K^{\prime}(x)=K(x)$ and $K \subseteq K^{\prime}$. Since $K^{\prime} \in \mathbb{C}_{2}$ and $K \in \operatorname{MD}\left(\mathbb{C}_{2}, x\right)$, it holds that $K=K^{\prime}$. Hence, $K \in \operatorname{MD}(\mathbb{C}, x)$.

3. Let $\mathbb{C}$ be finite, then $\mathbb{C}_{2}$ is a fuzzy subcovering of $\mathbb{C}_{\cap}$, and $x \in U$. If $K \in \operatorname{MD}(\mathbb{C}, x)$, then $K \in \mathbb{C}_{2} \subseteq \mathbb{C}_{\cap}$ and $K(x)>0$. Let $K^{\prime} \in \mathbb{C}_{\cap}$ with $K^{\prime}(x)=K(x)>0$ and $K \subseteq K^{\prime}$. Since $K^{\prime} \in \mathbb{C}$ and $K \in \operatorname{MD}(\mathbb{C}, x)$, it holds that $K=K^{\prime}$. Hence, $K \in \operatorname{MD}\left(\mathbb{C}_{\cap}, x\right)$.

On the other hand, if $K \in \operatorname{MD}\left(\mathbb{C}_{\cap}, x\right)$, then $K \in \mathbb{C}_{\cap} \subseteq \mathbb{C}$ and $K(x)>0$. Hence, there exists $K^{\prime} \in \operatorname{MD}(\mathbb{C}, x)$ with $K^{\prime}(x)=K(x)$ and $K \subseteq K^{\prime}$. Since $K^{\prime} \in \mathbb{C}_{2} \subseteq \mathbb{C}_{\cap}$ and $K \in \operatorname{MD}\left(\mathbb{C}_{\cap}, x\right)$, it holds that $K=K^{\prime}$. Hence, $K \in \operatorname{MD}(\mathbb{C}, x)$.

Note that in the fuzzy setting the equalities $\operatorname{md}\left(\mathbb{C}_{2}, x\right)=\mathscr{C}(\mathbb{C}, x)$ and $\operatorname{MD}\left(\mathbb{C}_{2}, x\right)=\mathscr{C}(\mathbb{C}, x)$ no longer hold as illustrated in the next example.

Example 7. Let $U=\{x, y\}$ and $\mathbb{C}=\left\{K_{1}, K_{2}\right\}$ with $K_{1}=1 / x+0.5 / y$ and $K_{2}=1 / x+1 / y$, then $\mathbb{C}_{2}=\mathbb{C}$. We have that $\mathscr{C}\left(\mathbb{C}_{2}, x\right)=\mathscr{C}(\mathbb{C}, x)=\left\{K_{1}, K_{2}\right\}, \operatorname{md}\left(\mathbb{C}_{2}, x\right)=\left\{K_{1}\right\}$ and $\operatorname{MD}\left(\mathbb{C}_{2}, x\right)=\left\{K_{2}\right\}$.

The first group we discuss, contains the fuzzy neighborhood operators $N_{1}^{\mathbb{C}}, N_{1}^{\mathbb{C}_{1}}, N_{1}^{\mathbb{C}_{3}}, N_{1}^{\mathbb{C}_{n}}$ and $N_{2}^{\mathbb{C}_{3}}$. We show that the first four operators are still equal in the fuzzy setting, but the last operator is different.

Proposition 14. Let $\mathbb{C}$ be a finite fuzzy covering and $\mathscr{I}$ an implicator used to define the covering $\mathbb{C}_{3}$ and the operators $N_{1}^{\mathbb{C}}, N_{1}^{\mathbb{C}_{1}}, N_{1}^{\mathbb{C}_{3}}$ and $N_{1}^{\mathbb{C}}$, then

1. $N_{1}^{\mathbb{C}}=N_{1}^{\mathbb{C}_{1}}$

2. $N_{1}^{\mathbb{C}}=N_{1}^{\mathbb{C}_{3}}$ if $\mathscr{I}$ is the R-implicator of a left-continuous $t$-norm,

3. $N_{1}^{\mathbb{C}}=N_{1}^{\mathbb{C}}$.

Proof. 1. This follows immediately from Proposition 3 and (1) of Proposition 13.

2. Assume that $\mathscr{I}$ is an R-implicator of a left-continuous t-norm $\mathscr{T}$. Since $N_{1}^{\mathbb{C}}(x) \in \mathbb{C}_{3}$, we have for $y \in U$ that $N_{1}^{\mathbb{C}_{3}}(x)(y) \leq \mathscr{I}\left(N_{1}^{\mathbb{C}}(x)(x), N_{1}^{\mathbb{C}}(x)(y)\right)=\mathscr{I}\left(1, N_{1}^{\mathbb{C}}(x)(y)\right)=N_{1}^{\mathbb{C}}(x)(y)$.

On the other hand, by Proposition $6, N_{1}^{\mathbb{C}}$ is $\mathscr{T}$-transitive. Therefore, for all $z \in U$, we have

$$
\begin{aligned}
\mathscr{T}\left(N_{1}^{\mathbb{C}}(z)(x), N_{1}^{\mathbb{C}}(x)(y)\right) \leq N_{1}^{\mathbb{C}}(z)(y) & \Rightarrow \mathscr{T}\left(N_{1}^{\mathbb{C}}(x)(y), N_{1}^{\mathbb{C}}(z)(x)\right) \leq N_{1}^{\mathbb{C}}(z)(y) \\
& \Rightarrow N_{1}^{\mathbb{C}}(x)(y) \leq \mathscr{I}\left(N_{1}^{\mathbb{C}}(z)(x), N_{1}^{\mathbb{C}}(z)(y)\right),
\end{aligned}
$$

hence, $N_{1}^{\mathbb{C}}(x)(y) \leq \inf _{z \in U} \mathscr{I}\left(N_{1}^{\mathbb{C}}(z)(x), N_{1}^{\mathbb{C}}(z)(y)\right)=\inf _{K \in \mathbb{C}_{3}} \mathscr{I}(K(x), K(y))=N_{1}^{\mathbb{C}_{3}}(x)(y)$.

3. Since $\mathbb{C}_{\cap} \subseteq \mathbb{C}, N_{1}^{\mathbb{C}}(x) \subseteq N_{1}^{\mathbb{C}}(x)$ for all $x \in U$.

On the other hand, take $y \in U$. Since $\mathbb{C}$ is finite, let $K \in \mathbb{C}$ such that $N_{1}^{\mathbb{C}}(x)(y)=\mathscr{I}(K(x), K(y))$. If $K \in \mathbb{C}_{\cap}$, then $N_{1}^{\mathbb{C} \cap}(x)(y) \leq \mathscr{I}(K(x), K(y))=N_{1}^{\mathbb{C}}(x)(y)$. If $K \notin \mathbb{C}_{\cap}$, we can find a collection $\mathbb{C}^{\prime} \subseteq \mathbb{C}_{\cap}$ such that $K=\bigcap \mathbb{C}^{\prime}$. Since $\mathbb{C}$ is finite, there exists $K^{\prime} \in \mathbb{C}^{\prime}$ with $K^{\prime}(y)=K(y)$ and $K \subseteq K^{\prime}$. Therefore, $\mathscr{I}(K(x), K(y)) \geq \mathscr{I}\left(K^{\prime}(x), K^{\prime}(y)\right)$. Moreover, we also have that $\mathscr{I}(K(x), K(y)) \leq \mathscr{I}\left(K^{\prime}(x), K^{\prime}(y)\right)$ since the infimum of $N_{1}^{\mathbb{C}}(x)(y)$ is reached in $K$ and $K^{\prime} \in \mathbb{C}$. Therefore, $N_{1}^{\mathbb{C} \cap}(x)(y) \leq \mathscr{I}\left(K^{\prime}(x), K^{\prime}(y)\right)=\mathscr{I}(K(x), K(y))=N_{1}^{\mathbb{C}}(x)(y)$. In both cases we can conclude that $N_{1}^{\mathbb{C}}(x) \subseteq N_{1}^{\mathbb{C}}(x)$. 
In the following example, we illustrate that the operators $N_{1}^{\mathbb{C}}$ and $N_{2}^{\mathbb{C}_{3}}$ are, in general, no longer equal.

Example 8. Let $U=\{x, y, z\}$ and $\mathbb{C}=\left\{K_{1}, K_{2}, K_{3}\right\}$ with $K_{1}=1 / x+0.8 / y+0.6 / z, K_{2}=0.2 / x+1 / y+0.6 / z$ and $K_{3}=0.2 / x+0.8 / y+1 / z$. Let $\mathscr{T}$ be the minimum operator and $\mathscr{I}$ its $R$-implicator defined by $\mathscr{I}(a, b)=1$ if $a \leq b$ and $\mathscr{I}(a, b)=b$ otherwise, then $N_{1}^{\mathbb{C}}(y)(x)=0.2$. On the other hand, $\mathbb{C}_{3}=\mathbb{C}$ and $N_{2}^{\mathbb{C}_{3}}(y)(x)=0.8$.

Next, we consider the group with operators $N_{2}^{\mathbb{C}}$ and $N_{2}^{\mathbb{C}_{1}}$. These operators are still equal in the fuzzy setting.

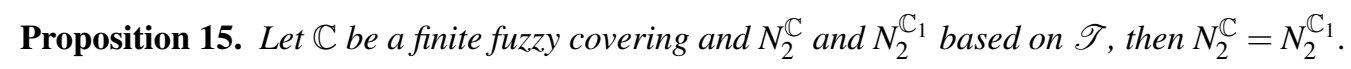

Proof. This follows immediately from (1) of Proposition 13.

The third group we discuss, is the group containing the operators $N_{3}^{\mathbb{C}}, N_{3}^{\mathbb{C}_{2}}, N_{3}^{\mathbb{C}}$ and $N_{1}^{\mathbb{C}_{2}}$. The first three fuzzy neighborhood operators are still equal, but the fourth operator is different in the fuzzy setting.

Proposition 16. Let $\mathbb{C}$ be a finite fuzzy covering $N_{3}^{\mathbb{C}}, N_{3}^{\mathbb{C}_{2}}$ and $N_{3}^{\mathbb{C}}$ based on $\mathscr{I}$, then

1. $N_{3}^{\mathbb{C}}=N_{3}^{\mathbb{C}_{2}}$,

2. $N_{3}^{\mathbb{C}}=N_{3}^{\mathbb{C}}$.

Proof. This follows immediately from (2) and (3) of Proposition 13.

The fuzzy neighborhood operators $N_{3}^{\mathbb{C}}$ and $N_{1}^{\mathbb{C}_{2}}$ are no longer equal.

Example 9. Let $U=\{x, y\}$ and $\mathbb{C}=\left\{K_{1}, K_{2}\right\}$ with $K_{1}=1 / x+0.5 / y$ and $K_{2}=1 / x+1 / y$, then $\mathbb{C}_{2}=\mathbb{C}$. Let $\mathscr{I}$ be a border implicator, i.e., $\mathscr{I}(1, a)=$ a for all $a \in[0,1]$. We have that $N_{3}^{\mathbb{C}}(x)(y)=\mathscr{I}\left(K_{2}(x), K_{2}(y)\right)=1$ and $N_{1}^{\mathbb{C}_{2}}(x)(y)=$ $\mathscr{I}\left(K_{1}(x), K_{1}(y)\right)=0.5$.

The final group we discuss consists of the operators $N_{4}^{\mathbb{C}}, N_{4}^{\mathbb{C}_{2}}, N_{4}^{\mathbb{C}}$ and $N_{2}^{\mathbb{C}_{2}}$. The first three operators are equal, but the fourth operator is different in the fuzzy setting.

Proposition 17. Let $\mathbb{C}$ be a finite fuzzy covering and $N_{4}^{\mathbb{C}}, N_{4}^{\mathbb{C}_{2}}$ and $N_{4}^{\mathbb{C}}$ based on $\mathscr{T}$, then

1. $N_{4}^{\mathbb{C}}=N_{4}^{\mathbb{C}_{2}}$,

2. $N_{4}^{\mathbb{C}}=N_{4}^{\mathbb{C}}$.

Proof. This follows immediately from Proposition 4 and (2) and (3) of Proposition 13.

The fuzzy neighborhood operators $N_{4}^{\mathbb{C}}$ and $N_{2}^{\mathbb{C}_{2}}$ are no longer equal.

Example 10. Let $U=\{x, y\}$ and $\mathbb{C}=\left\{K_{1}, K_{2}\right\}$ with $K_{1}=1 / x+0.5 / y$ and $K_{2}=1 / x+1 / y$, then $\mathbb{C}_{2}=\mathbb{C}$. Let $\mathscr{T}$ be t-norm. We have that $N_{4}^{\mathbb{C}}(x)(y)=\mathscr{T}\left(K_{2}(x), K_{2}(y)\right)=1$ and $N_{2}^{\mathbb{C}_{2}}(x)(y)=\mathscr{T}\left(K_{1}(x), K_{1}(y)\right)=0.5$.

We conclude that given a finite fuzzy covering $\mathbb{C}$, a left-continuous t-norm to construct $\mathbb{C}_{4}$ and the operators $N_{2}^{\mathbb{C}_{j}}$ and $N_{4}^{\mathbb{C}_{j}}$ and its R-implicator to construct $\mathbb{C}_{3}$ and the operators $N_{1}^{\mathbb{C}_{j}}$ and $N_{3}^{\mathbb{C}_{j}}$, we have sixteen different groups of fuzzy neighborhood operators, listed in Table 2.

In the next section, we describe the partial order relations between the different groups of fuzzy neighborhood operators of Table 2 . 
Table 2: Fuzzy neighborhood operators based on fuzzy coverings

\begin{tabular}{ll|ll}
\hline Group & Operators & Group & Operators \\
\hline$A 1$. & $N_{1}^{\mathbb{C}}, N_{1}^{\mathbb{C}_{1}}, N_{1}^{\mathbb{C}_{3}}, N_{1}^{\mathbb{C}_{n}}$ & G. & $N_{1}^{\mathbb{C}_{4}}$ \\
\hline$A 2$. & $N_{2}^{\mathbb{C}_{3}}$ & H. & $N_{4}^{\mathbb{C}}, N_{4}^{\mathbb{C}_{2}}, N_{4}^{\mathbb{C}_{n}}$ \\
\hline$B$. & $N_{3}^{\mathbb{C}_{1}}$ & H. & $N_{2}^{\mathbb{C}_{2}}$ \\
\hline$C$. & $N_{3}^{\mathbb{C}_{3}}$ & I. & $N_{2}^{\mathbb{C}_{4}}$ \\
\hline$D$. & $N_{4}^{\mathbb{C}_{3}}$ & J. & $N_{3}^{\mathbb{C}_{4}}$ \\
\hline$E$. & $N_{2}^{\mathbb{C}}, N_{2}^{\mathbb{C}_{1}}$ & $K$. & $N_{4}^{\mathbb{C}_{4}}$ \\
\hline$F 1$. & $N_{3}^{\mathbb{C}}, N_{3}^{\mathbb{C}_{2}}, N_{3}^{\mathbb{C}_{n}}$ & $L$. & $N_{4}^{\mathbb{C}_{1}}$ \\
\hline$F 2$. & $N_{1}^{\mathbb{C}_{2}}$ & $M$. & $N_{2}^{\mathbb{C}_{n}}$ \\
\hline
\end{tabular}

\section{A lattice of fuzzy neighborhood operators based on a finite fuzzy covering}

In this section, we assume $\mathbb{C}$ to be a finite fuzzy covering, $\mathscr{T}$ a left-continuous t-norm which is used to define the covering $\mathbb{C}_{4}$ and the operators $N_{2}^{\mathbb{C}_{j}}$ and $N_{4}^{\mathbb{C}_{j}}$ and $\mathscr{I}$ its R-implicator which is used to define $\mathbb{C}_{3}$ and the operators $N_{1}^{\mathbb{C}_{j}}$ and $N_{3}^{\mathbb{C}_{j}}$, in order to guarantee all equalities of Table 2 .

We define a partial order relation $\leq$ between fuzzy neighborhood operators as follows: $N \leq N^{\prime}$ if and only if $\forall x, y \in U: N(x)(y) \leq N^{\prime}(x)(y)$. We say that two fuzzy neighborhood operators $N$ and $N^{\prime}$ are incomparable if neither $N \leq N^{\prime}$ nor $N^{\prime} \leq N$ hold. Note that if two crisp neighborhood operators are incomparable, their fuzzy extensions are incomparable as well, e.g., the fuzzy neighborhood operators $N_{3}^{\mathbb{C}_{1}}$ (group $B$ ) and $N_{3}^{\mathbb{C}_{3}}$ (group $C$ ) are incomparable. Therefore, we only need to consider the partial order relations given in Figure 1.

In [20] it was proved that for each crisp covering $\mathbb{C}$ it holds that $N_{1}^{\mathbb{C}} \leq N_{2}^{\mathbb{C}} \leq N_{4}^{\mathbb{C}}$ and $N_{1}^{\mathbb{C}} \leq N_{3}^{\mathbb{C}} \leq N_{4}^{\mathbb{C}}$. These relationships are maintained in the fuzzy setting.

Proposition 18. Let $\mathbb{C}$ be a finite fuzzy covering, $\mathscr{T}$ a left-continuous t-norm used to define the operators $N_{2}^{\mathbb{C}}$ and $N_{4}^{\mathbb{C}}$ and $\mathscr{I}$ its $R$-implicator used to define the operators $N_{1}^{\mathbb{C}}$ and $N_{3}^{\mathbb{C}}$, then

1. $N_{1}^{\mathbb{C}} \leq N_{2}^{\mathbb{C}}$,

2. $N_{1}^{\mathbb{C}} \leq N_{3}^{\mathbb{C}}$,

3. $N_{2}^{\mathbb{C}} \leq N_{4}^{\mathbb{C}}$,

4. $N_{3}^{\mathbb{C}} \leq N_{4}^{\mathbb{C}}$.

Proof. 1. Assume that the inclusion does not hold for $x, y \in U$, then

$$
\sup _{K \in \operatorname{md}(\mathbb{C}, x)} \mathscr{T}(K(x), K(y))<\inf _{K \in \mathbb{C}} \mathscr{I}(K(x), K(y)),
$$

i.e., for all $K_{1} \in \operatorname{md}(\mathbb{C}, x)$ and for all $K_{2} \in \mathbb{C}$ it holds that $\mathscr{T}\left(K_{1}(x), K_{1}(y)\right)<\mathscr{I}\left(K_{2}(x), K_{2}(y)\right)$. Take $K^{*} \in \mathbb{C}$ such that $K^{*}(x)=1$ and take $K^{\prime} \in \operatorname{md}(\mathbb{C}, x)$ such that $K^{\prime}(x)=K^{*}(x)=1$ and $K^{\prime} \subseteq K^{*}$. Then for $K_{1}=K_{2}=K^{\prime}$ we have $\mathscr{T}\left(K^{\prime}(x), K^{\prime}(y)\right)<\mathscr{I}\left(K^{\prime}(x), K^{\prime}(y)\right)$, hence, $K^{\prime}(y)<K^{\prime}(y)$, which is a contradiction.

2. This follows immediately from the fact that $\operatorname{MD}(\mathbb{C}, x) \subseteq \mathbb{C}$.

3. This follows immediately from the fact that $\operatorname{md}(\mathbb{C}, x) \subseteq \mathbb{C}$.

4. Assume that the inclusion does not hold for $x, y \in U$, then

$$
\sup _{K \in \mathbb{C}} \mathscr{T}(K(x), K(y))<\inf _{K \in \mathrm{MD}(\mathbb{C}, x)} \mathscr{I}(K(x), K(y))
$$


i.e., for all $K_{1} \in \mathbb{C}$ and for all $K_{2} \in \operatorname{MD}(\mathbb{C}, x)$ it holds that $\mathscr{T}\left(K_{1}(x), K_{1}(y)\right)<\mathscr{I}\left(K_{2}(x), K_{2}(y)\right)$. Take $K^{*} \in \mathbb{C}$ such that $K^{*}(x)=1$ and take $K^{\prime} \in \operatorname{MD}(\mathbb{C}, x)$ such that $K^{\prime}(x)=K^{*}(x)=1$ and $K^{*} \subseteq K^{\prime}$. Then for $K_{1}=K_{2}=K^{\prime}$ we have $\mathscr{T}\left(K^{\prime}(x), K^{\prime}(y)\right)<\mathscr{I}\left(K^{\prime}(x), K^{\prime}(y)\right)$, hence, $K^{\prime}(y)<K^{\prime}(y)$, which is a contradiction.

Note that (1) and (4) of Proposition 18 uses Proposition 2, while the partial order relations in (2) and (3) also hold for an infinite $\mathbb{C}$. Proposition 18 implies that the following partial order relations hold for Table 2.

Corollary 1. Let $\mathbb{C}$ be a finite fuzzy covering, $\mathscr{T}$ a left-continuous $t$-norm used to define the covering $\mathbb{C}_{4}$ and the operators $N_{2}^{\mathbb{C}_{j}}$ and $N_{4}^{\mathbb{C}_{j}}$ and $\mathscr{I}$ its $R$-implicator used to define the covering $\mathbb{C}_{3}$ and the operators $N_{1}^{\mathbb{C}_{j}}$ and $N_{3}^{\mathbb{C}_{j}}$, then

1. for $\mathbb{C}$ we obtain that $A 1 \leq E \leq H 1$ and $A 1 \leq F 1 \leq H 1$,

2. for $\mathbb{C}_{1}$ we obtain that $A 1 \leq E \leq L$ and $A 1 \leq B \leq L$,

3. for $\mathbb{C}_{2}$ we obtain that $F 2 \leq H 2 \leq H 1$ and $F 2 \leq F 1 \leq H 1$,

4. for $\mathbb{C}_{3}$ we obtain that $A 1 \leq A 2 \leq D$ and $A 1 \leq C \leq D$,

5. for $\mathbb{C}_{4}$ we obtain that $G \leq I \leq \bar{K}$ and $G \leq J \leq K$,

6. for $\mathbb{C}_{\cap}$ we obtain that $A 1 \leq \bar{M} \leq H 1$ and $A 1 \leq F 1 \leq H 1$.

Moreover, since $\mathbb{C}_{1}$ and $\mathbb{C}_{2}$ are subcoverings of the finite fuzzy covering $\mathbb{C}$, we obtain that $A 1 \leq F 2$ and $L \leq H 1$.

Proposition 19. Let $\mathbb{C}$ be a finite fuzzy covering, $\mathscr{T}$ a left-continuous $t$-norm used to define $N_{4}^{\mathbb{C}}$ and $N_{4}^{\mathbb{C}_{1}}$ and $\mathscr{I}$ its $R$-implicator used to define $N_{1}^{\mathbb{C}}$ and $N_{1}^{\mathbb{C}_{1}}$, then $N_{1}^{\mathbb{C}} \leq N_{1}^{\mathbb{C}_{2}}$ and $N_{4}^{\mathbb{C}_{1}} \leq N_{4}^{\mathbb{C}}$.

Proof. This follows immediately from $\mathbb{C}_{2} \subseteq \mathbb{C}$ and $\mathbb{C}_{1} \subseteq \mathbb{C}$.

Furthermore, it holds that $F 1 \leq G \leq H 1 \leq I$. To prove these partial order relations, we first consider the following lemma.

Lemma 2. [19] Let $\mathscr{T}$ a left-continuous t-norm and $\mathscr{I}$ its R-implicator, then $\mathscr{I}(a, b) \leq \mathscr{I}(\mathscr{T}(a, c), \mathscr{T}(c, b))$ holds for all $a, b, c \in[0,1]$.

Proposition 20. Let $\mathbb{C}$ be a finite fuzzy covering, $\mathscr{T}$ a left-continuous t-norm used to define the covering $\mathbb{C}_{4}$ and the operators $N_{4}^{\mathbb{C}}$ and $N_{2}^{\mathbb{C}_{4}}$ and $\mathscr{I}$ its $R$-implicator used to define the operators $N_{3}^{\mathbb{C}}$ and $N_{1}^{\mathbb{C}_{4}}$, then

1. $N_{3}^{\mathbb{C}} \leq N_{1}^{\mathbb{C}_{4}}$,

2. $N_{1}^{\mathbb{C}_{4}} \leq N_{4}^{\mathbb{C}}$,

3. $N_{4}^{\mathbb{C}} \leq N_{2}^{\mathbb{C}_{4}}$.

Proof. 1. Let $x, y \in U$, then

$$
\begin{aligned}
& N_{1}^{\mathbb{C}_{4}}(x)(y)=\inf _{z \in U} \mathscr{I}\left(N_{4}^{\mathbb{C}}(z)(x), N_{4}^{\mathbb{C}}(z)(y)\right) \\
& =\inf _{z \in U} \mathscr{I}\left(N_{4}^{\mathbb{C}}(x)(z), N_{4}^{\mathbb{C}}(z)(y)\right) \\
& =\inf _{z \in U} \mathscr{I}\left(\sup _{K \in \mathrm{MD}(\mathbb{C}, x)} \mathscr{T}(K(x), K(z)), N_{4}^{\mathbb{C}}(z)(y)\right)
\end{aligned}
$$

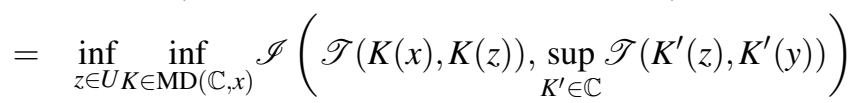

$$
\begin{aligned}
& \geq \inf _{z \in U} \inf _{K \in \mathrm{MD}(\mathbb{C}, x)} \sup _{K^{\prime} \in \mathbb{C}} \mathscr{I}\left(\mathscr{T}(K(x), K(z)), \mathscr{T}\left(K^{\prime}(z), K^{\prime}(y)\right)\right)
\end{aligned}
$$

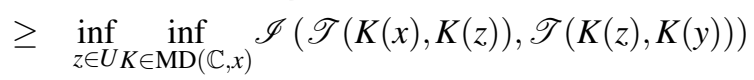

$$
\begin{aligned}
& =\inf _{K \in \mathrm{MD}(\mathbb{C}, x)} \inf _{z \in U} \mathscr{I}(\mathscr{T}(K(x), K(z)), \mathscr{T}(K(z), K(y))) \\
& \geq \inf _{K \in \mathrm{MD}(\mathbb{C}, x)} \mathscr{I}(K(x), K(y)) \\
& =N_{3}^{\mathbb{C}}(x)(y)
\end{aligned}
$$


where in the penultimate step we have used Lemma 2.

2. Let $x, y \in U$, then $N_{1}^{\mathbb{C}_{4}}(x)(y) \leq \mathscr{I}\left(N_{4}^{\mathbb{C}}(x)(x), N_{4}^{\mathbb{C}}(x)(y)\right)=\mathscr{I}\left(1, N_{4}^{\mathbb{C}}(x)(y)\right)=N_{4}^{\mathbb{C}}(x)(y)$.

3. Let $x, y \in U$. If $N_{4}^{\mathbb{C}}(x) \in \operatorname{md}\left(\mathbb{C}_{4}, x\right)$, then we have that

$$
N_{2}^{\mathbb{C}_{4}}(x)(y) \geq \mathscr{T}\left(N_{4}^{\mathbb{C}}(x)(x), N_{4}^{\mathbb{C}}(x)(y)\right)=\mathscr{T}\left(1, N_{4}^{\mathbb{C}}(x)(y)\right)=N_{4}^{\mathbb{C}}(x)(y) .
$$

Similarly, if $N_{4}^{\mathbb{C}}(y) \in \operatorname{md}\left(\mathbb{C}_{4}, x\right)$, then $N_{2}^{\mathbb{C}_{4}}(x)(y) \geq \mathscr{T}\left(N_{4}^{\mathbb{C}}(y)(x), N_{4}^{\mathbb{C}}(y)(y)\right)=N_{4}^{\mathbb{C}}(x)(y)$. Now assume that neither $N_{4}^{\mathbb{C}}(x)$ nor $N_{4}^{\mathbb{C}}(y)$ belong to $\operatorname{md}\left(\mathbb{C}_{4}, x\right)$. Hence,

$$
\begin{aligned}
& \exists z_{1} \in U: N_{4}^{\mathbb{C}}\left(z_{1}\right)(x)=N_{4}^{\mathbb{C}}(x)(x)=1, N_{4}^{\mathbb{C}}\left(z_{1}\right) \subseteq N_{4}^{\mathbb{C}}(x), \\
& \exists z_{2} \in U: N_{4}^{\mathbb{C}}\left(z_{2}\right)(x)=N_{4}^{\mathbb{C}}(y)(x), N_{4}^{\mathbb{C}}\left(z_{2}\right) \subseteq N_{4}^{\mathbb{C}}(y),
\end{aligned}
$$

with $N_{4}^{\mathbb{C}}\left(z_{1}\right), N_{4}^{\mathbb{C}}\left(z_{2}\right) \in \operatorname{md}\left(\mathbb{C}_{4}, x\right)$. Note that $N_{4}^{\mathbb{C}}(y)\left(z_{2}\right) \geq N_{4}^{\mathbb{C}}\left(z_{2}\right)\left(z_{2}\right)=1$. We derive that

$$
\begin{aligned}
N_{2}^{\mathbb{C}_{4}}(x)(y) & \geq \max \left(\mathscr{T}\left(N_{4}^{\mathbb{C}}\left(z_{1}\right)(x), N_{4}^{\mathbb{C}}\left(z_{1}\right)(y)\right), \mathscr{T}\left(N_{4}^{\mathbb{C}}\left(z_{2}\right)(x), N_{4}^{\mathbb{C}}\left(z_{2}\right)(y)\right)\right) \\
& =\max \left(\mathscr{T}\left(1, N_{4}^{\mathbb{C}}\left(z_{1}\right)(y)\right), \mathscr{T}\left(N_{4}^{\mathbb{C}}(y)(x), 1\right)\right) \\
& =\max \left(N_{4}^{\mathbb{C}}\left(z_{1}\right)(y), N_{4}^{\mathbb{C}}(x)(y)\right) \\
& =N_{4}^{\mathbb{C}}(x)(y) .
\end{aligned}
$$

Furthermore, we can prove that $D \leq L$.

Proposition 21. Let $\mathbb{C}$ be a finite fuzzy covering, $\mathscr{T}$ a left-continuous t-norm used to define the operators $N_{4}^{\mathbb{C}_{3}}$ and $N_{4}^{\mathbb{C}_{1}}$ and $\mathscr{I}$ its R-implicator used to define the covering $\mathbb{C}_{3}$, then $N_{4}^{\mathbb{C}_{3}} \leq N_{4}^{\mathbb{C}_{1}}$.

Proof. Let $x, y, z \in U$ and let $K^{*} \in \operatorname{md}(\mathbb{C}, z)$ with $K^{*}(z)=1$, then

$$
\begin{aligned}
\mathscr{T}\left(N_{1}^{\mathbb{C}}(z)(x), N_{1}^{\mathbb{C}}(z)(x)\right) & \leq \inf _{K \in \operatorname{md}(\mathbb{C}, z)} \mathscr{T}(\mathscr{I}(K(z), K(x)), \mathscr{I}(K(z), K(y))) \\
& \leq \mathscr{T}\left(\mathscr{I}\left(K^{*}(z), K^{*}(x)\right), \mathscr{I}\left(K^{*}(z), K^{*}(y)\right)\right) \\
& =\mathscr{T}\left(\mathscr{I}\left(1, K^{*}(x)\right), \mathscr{I}\left(1, K^{*}(y)\right)\right) \\
& =\mathscr{T}\left(K^{*}(x), K^{*}(y)\right) \\
& \leq \sup _{K \in \operatorname{md}(\mathbb{C}, z)} \mathscr{T}(K(x), K(y)) .
\end{aligned}
$$

Hence, we obtain that

$$
\begin{aligned}
& N_{4}^{\mathbb{C}_{3}}(x)(y)=\sup _{z \in U} \mathscr{T}\left(N_{1}^{\mathbb{C}}(z)(x), N_{1}^{\mathbb{C}}(z)(x)\right) \\
& \leq \sup _{z \in U K \in \operatorname{md}(\mathbb{C}, z)} \sup _{\mathscr{T}} \mathscr{T}(K(x), K(y)) \\
& =\sup _{K \in \mathbb{C}_{1}} \mathscr{T}(K(x), K(y)) \\
& =N_{4}^{\mathbb{C}_{1}}(x)(y) \text {. }
\end{aligned}
$$

To end, we show that $M \leq H 2$.

Proposition 22. Let $\mathbb{C}$ be a finite fuzzy covering and $\mathscr{T}$ a left-continuous t-norm used to define the operators $N_{2}^{\mathbb{C}_{n}}$ and $N_{2}^{\mathbb{C}_{2}}$, then $N_{2}^{\mathbb{C}_{n}} \leq N_{2}^{\mathbb{C}_{2}}$. 
Proof. Let $x \in U$, then we first prove that $\operatorname{md}\left(\mathbb{C}_{\cap}, x\right) \cap \mathbb{C}_{2} \subseteq \operatorname{md}\left(\mathbb{C}_{2}, x\right)$. Let $K \in \operatorname{md}\left(\mathbb{C}_{\cap}, x\right) \cap \mathbb{C}_{2}$ and take $K^{\prime} \in \mathbb{C}_{2}$ with $K^{\prime}(x)=K(x)$ and $K^{\prime} \subseteq K$. As $K^{\prime} \in \mathbb{C}_{\cap}$ and $K \in \operatorname{md}\left(\mathbb{C}_{\cap}, x\right)$, we obtain $K=K^{\prime}$ and thus, $K \in \operatorname{md}\left(\mathbb{C}_{2}, x\right)$.

Let $y \in U$ and assume that $N_{2}^{\mathbb{C}_{\cap}}(x)(y)=\mathscr{T}\left(K^{*}(x), K^{*}(y)\right)$ for $K^{*} \in \operatorname{md}\left(\mathbb{C}_{\cap}, x\right)$. If $K^{*} \notin \mathbb{C}_{2}$, then for each $z \in U$ there exists a $K_{z} \in \mathbb{C}_{2}$ such that $K^{*}(z)=K_{z}(z)$ and $K^{*} \subsetneq K_{z}$. However, this means that $K^{*}=\bigcap_{z \in U} K_{z}$. As $K^{*} \in \mathbb{C}_{\cap}$, this is a contradiction. Therefore, $K^{*}$ will be a set in $\mathbb{C}_{2}$ and by the observation above, $K^{*} \in \operatorname{md}\left(\mathbb{C}_{2}, x\right)$ is obtained and thus, $N_{2}^{\mathbb{C} \cap}(x)(y) \leq N_{2}^{\mathbb{C}_{2}}(x)(y)$.

There are no other comparable operators, i.e., the fuzzy neighborhood operator $N_{2}^{\mathbb{C}_{3}}$ (group $A 2$ ) is not less or equal than the fuzzy neighborhood operators of the groups $B, C, E, F 1, F 2, G, H 2, J$ and $M$ with respect to the partial order relation $\leq$, and therefore, $A 2$ is incomparable with them. Moreover the fuzzy neighborhood operator $N_{2}^{\mathbb{C}_{2}}$ (group $H 2$ ) is not greater or equal than the fuzzy neighborhood operators of the groups $B, C, D, E, F 1, G$ and $L$ with respect to the partial order relation $\leq$, and thus, $H 2$ is incomparable with them. We illustrate this in the following two examples.

Example 11. Let $\mathscr{T}$ be the product $t$-norm defined by $\mathscr{T}(a, b)=a \cdot b$ for all $a, b \in[0,1]$ and $\mathscr{I}$ its $R$-implicator. Let $U=\{x, y, z\}$ and $\mathbb{C}=\left\{K_{1}, K_{2}, K_{3}, K_{4}\right\}$ with $K_{1}=1 / x+0.2 / y+0.6 / z, K_{2}=0.5 / x+1 / y+0.6 / z, K_{3}=0.5 / x+0.6 / y+$ $1 / z$ and $K_{4}=0.5 / x+0.5 / y+0 / z$. Then we have that

- $N_{2}^{\mathbb{C}_{3}}(x)(y)=0.5($ group $A 2)$,

- $N_{2}^{\mathbb{C}}(x)(y)=N_{2}^{\mathbb{C}_{2}}(x)(y)=N_{2}^{\mathbb{C}_{n}}(x)(y)=0.25$ (groups $\left.E, H 2, M\right)$,

- $N_{3}^{\mathbb{C}_{1}}(x)(y)=N_{3}^{\mathbb{C}_{3}}(x)(y)=N_{3}^{\mathbb{C}}(x)(y)=N_{1}^{\mathbb{C}_{2}}(x)(y)=0.2$ (groups B, C, F1, F2).

Hence, $A 2$ is incomparable with the fuzzy neighborhood operators of the groups $B, C, E, F 1, F 2, H 2$ and $M$.

On the other hand, let $\mathbb{C}=\left\{K_{1}, K_{2}, K_{3}, K_{4}\right\}$ with $K_{1}=1 / x+0.8 / y+0.6 / z, K_{2}=0.2 / x+1 / y+0.6 / z, K_{3}=$ $0.2 / x+0.8 / y+1 / z$ and $K_{4}=0.1 / x+0.6 / y+1 / z$ and let $\mathscr{T}$ be the minimum operator and $\mathscr{I}$ its $R$-implicator. Then we have that $N_{2}^{\mathbb{C}_{3}}(y)(x)=0.8$ and $N_{1}^{\mathbb{C}_{4}}(y)(x)=N_{3}^{\mathbb{C}_{4}}(y)(x)=0.6$, hence, $A 2$ is incomparable with $G$ and $J$.

Example 12. Let $U=\{x, y, z\}$ with $\mathbb{C}$ as in Example 4, $\mathscr{T}$ the minimum operator and $\mathscr{I}$ its $R$-impliator, then $N_{2}^{\mathbb{C}_{2}}(x)(y)=0.1$ and $N_{3}^{\mathbb{C}_{1}}(x)(y)=N_{4}^{\mathbb{C}_{3}}(x)(y)=N_{2}^{\mathbb{C}}(x)(y)=N_{3}^{\mathbb{C}}(x)(y)=N_{1}^{\mathbb{C}_{4}}(x)(y)=0.9$, therefore, H2 is incomparable with the groups $B, D, E, F 1$ and $G$.

On the other hand, let $\mathbb{C}=\left\{K_{1}, K_{2}, K_{3}, K_{4}\right\}$ with $K_{1}=1 / x+0.2 / y+0.6 / z, K_{2}=0.5 / x+1 / y+0.6 / z, K_{3}=0.5 / x+$ $0.6 / y+1 / z$ and $K_{4}=0.5 / x+0.5 / y+0 / z$ and $\mathscr{T}$ the product $t$-norm and $\mathscr{I}$ its $R$-implicator, then $N_{2}^{\mathbb{C}_{2}}(x)(y)=0.25$ and $N_{4}^{\mathbb{C}_{1}}(x)(y)=0.5$. Hence, H2 is incomparable with $L$.

To end, let $U=\{x, y\}$ and $\mathbb{C}=\left\{K_{1}, K_{2}\right\}$ with $K_{1}=1 / x+0.5 / y$ and $K_{2}=1 / x+1 / y$ and let $\mathscr{T}$ be the minimum operator and $\mathscr{I}$ its $R$-implicator, then $N_{2}^{\mathbb{C}_{2}}(x)(y)=0.5$ while $N_{3}^{\mathbb{C}_{3}}(x)(y)=1$. Hence, H2 is incomparable with $C$.

The results obtained in this section are summarized in Figure 2, where the partial order $\leq$ is shown to define a lattice on the set of fuzzy neighborhood operators of Table 2.

\section{Conclusion and future work}

In this article, given a fuzzy covering, we have introduced fuzzy extensions of the neighborhood system, the minimal description and maximal description of an object of the universe. Moreover, four crisp neighborhood operators and six crisp coverings studied in [28] are extended to the fuzzy setting, and in addition, some results concerning crisp neighborhood operators and crisp coverings are proven to be maintained. For a finite fuzzy covering, the four fuzzy neighborhood operators and six fuzzy coverings, one original and five derived ones, result in twenty-four combinations of fuzzy neighborhood operators. However, we have proven that for a left-continuous t-norm and its residual implicator the obtained twenty-four combinations can be reduced to sixteen different groups of fuzzy neighborhood operators. In this setting, we have obtained the Hasse diagram of these sixteen groups, which expresses which operators yield larger or smaller fuzzy neighborhoods. 


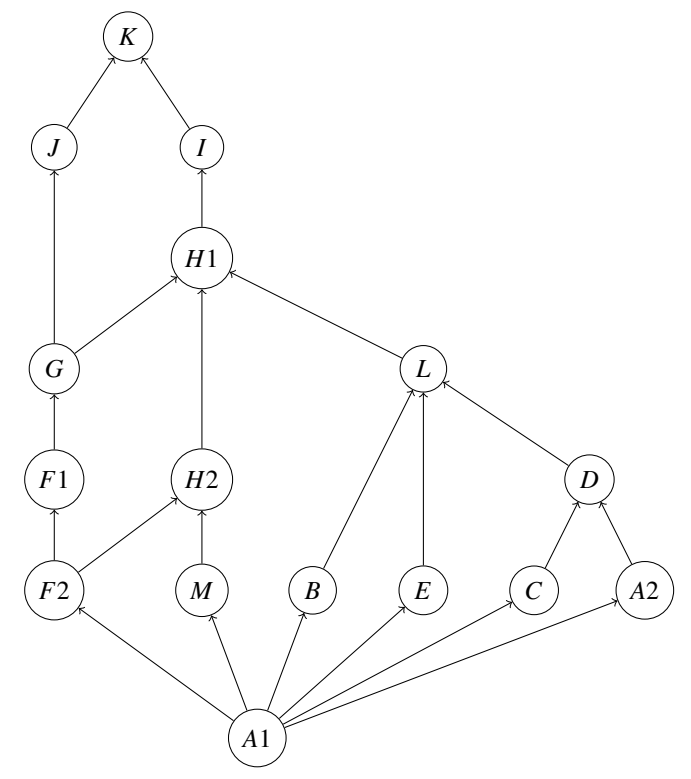

An essential future research direction is the application of these fuzzy neighborhood operators based on fuzzy coverings in fuzzy rough set models, as well as their practicality in data mining techniques such as feature selection $[3,8,11]$. Each fuzzy neighborhood operator presented in this work gives rise to a fuzzy approximation operator. The goal is to establish a framework combining those fuzzy approximation operators with the operators presented in, inter alia, [4, 5, 24].

However, it is crucial to define meaningful coverings based on the given data, often presented in an information table. As the results presented in this work were achieved from a computational approach to fuzzy covering-based rough sets, i.e., the focus of the research is on the construction of different rough set models, there is still a gap in the research field on rough sets of a conceptual and semantical understanding for (fuzzy) covering-based rough set models, as recently discussed by Yao [29]. In the latter approach, the focus is rather on the insight of concepts instead of providing computationally efficient algorithms. Hence, the study of (fuzzy) neighborhood operators based on coverings from a semantically sound approach will be a major topic in our future research directives as it will provide more motivation for the use of the different fuzzy neighborhood operators based on fuzzy coverings.

\section{Acknowledgements}

Lynn D'eer was supported by the Ghent University Special Research Fund. Chris Cornelis was partially supported by the Spanish Ministry of Science and Technology under the Project TIN2014-57251-P and the Andalusian Research Plans P10-TIC-6858, P11-TIC-7765 and P12-TIC-2958, and by Project PYR-2014-8 of the Genil Program of CEI BioTic GRANADA. Lluis Godo was partially supported by the Spanish MINECO project EdeTRI TIN2012-39348C02-01 and the network TIN2014-56381- REDTLODISCO, as well as the Catalan government grant 2014SGR-118.

\section{References}

[1] M. Baczyński, B. Jayaram, Fuzzy implications, Springer-Verlag Berlin, 2008

[2] Z. Bonikowski, E. Brynarski, Extensions and intensions in rough set theory, Information Sciences 107 (1998) 149-167

[3] C. Cornelis, R. Jensen, G. Hurtado Martín, D. Ślęzak, Attribute selection with fuzzy decision reducts, Information Sciences, 180 (16) (2010) 3006-3020

[4] L. D'eer, N. Verbiest, C. Cornelis, L. Godo, A comprehensive study of implicator-conjunctor-based and noise-tolerant fuzzy rough sets: definitions, properties and robustness analysis, Fuzzy Sets and Systems 275 (2015) 1-38 
[5] L. D'eer, C. Cornelis, D. Sánchez, Fuzzy covering based rough sets revisited, Proceedings of the 2015 Conference of the International Fuzzy Systems Association and the European Society for Fuzzy Logic and Technology (IFSA-EUSFLAT 2015), AISR, ISSN 1951-6851, volume 89 (2015) 651-658

[6] L. D'eer, M. Restrepo, C. Cornelis, J. Gómez, Neighborhood operators for covering based rough sets, Information Sciences 336 (2016) 21-44

[7] T. Deng, Y. Chen, W. Xu and Q. Dai, A novel approach to fuzzy rough sets based on a fuzzy covering, Information Sciences 177 (11) (2007) 2308-2326

[8] Y. Du, Q. Hu, D. Chen, P. Ma, Kernelized fuzzy rough sets based yawn detection for driver fatigue monitoring, Fundamenta Informaticae 111 (1) (2011) 65-79.

[9] T. Feng, S.P. Zhang, J.S. Mi, The reduction and fusion of fuzzy covering systems based on the evidence theory, International Journal of Approximate Reasoning 53 (2012) 87-103

[10] M. Inuiguchi, Classification and approximation oriented fuzzy rough sets, in B. Bouchon-Meunier et al. (eds.), Proceedings of Information Processing and Managment of Uncertainty in Knowledge-based Systems (2004) cd-rom

[11] R. Jensen, Q. Shen, Fuzzy-rough attribute reduction with application to web categorization, Fuzzy Sets and Systems 141 (3) (2004) $469-485$.

[12] E. E. Kerre and P. L. Ottoy, Lattice properties of neighborhood systems in Chang fuzzy topological spaces, Fuzzy Sets and Systems 30 (1989) 205-213

[13] T.J. Li, Y. Leung, W.X. Zhang, Generalized fuzzy rough approximation operators based on fuzzy coverings, International Journal of Approximate Reasoning 48 (2008) 836-856

[14] T. Y. Lin, Neigbhorhood systems: a qualitative theory for fuzzy and rough sets, University of California, Berkely 94720 (2007)

[15] Y. M. Liu and M. K. Luo, Fuzzy topology, Advances in Fuzzy Systems, Applications and Theory 9 (1997)

[16] R. Lowen, Fuzzy neigborhood spaces, Fuzzy Sets and Systems 7 (2) (1982) 165 - 189

[17] Z. Pawlak, Rough sets, International Journal of Computer and Information Sciences 11 (1982) 341-356

[18] J.A. Pomykala, Approximation operations in approximation space, Bulletin de la Académie Polonaise des Sciences 35 (9-10), (1987) 653662.

[19] A. M. Radzikowska and E. E. Kerre. Characterisation of main classes of fuzzy relations using fuzzy modal operators. Fuzzy Sets and Systems, 152 (223-247) 2005.

[20] M. Restrepo, C. Cornelis, J. Gómez, Partial order relation for approximation operators in covering based rough sets, Information Sciences 284 (2014) 44-59.

[21] R. Słowinski, D. Vanderpooten, A generalized definition of rough approximation based on similarity, IEEE Transaction on Knowledge and data Engineering 12 (2000) 331-336.

[22] M.F. Wu, H.H. Han, Y.F. Si, Properties and axiomatization of fuzzy rough sets based on fuzzy coverings, Proceedings of the 2012 International Conference on Machine Learning and Cybernetics (2012) 223-247

[23] W. Z. Wu, W. X. Zhang, Neighborhood operators systems and approximations, Information Sciences 144 (2002) $201-207$.

[24] W. Z. Wu, Y. Leung, M. W. Shao, Generalized fuzzy rough approximation operators determined by fuzzy implicators, International Journal of Approximate Reasoning 54 (2013) 1388-1409

[25] T. Yang, Q. Li, Reduction about approximation spaces of covering generalized rough sets. International Journal of Approximate Reasoning, 51 (2010) 335-345.

[26] Y. Y. Yao, Two views of the theory of rough sets in finite universes, International Journal of Approximate Reasoning 15 (4) (1996) $291-317$

[27] Y. Y. Yao, Relational interpretations of neighborhood operators and rough set approximation operators, Information Sciences 101 (1998) $21-47$

[28] Y. Y. Yao, B. Yao, Covering based rough sets approximations. Information Sciences 200 (2012) 91-107.

[29] Y. Y. Yao, The two sides of the theory of rough sets, Knowledge-Based Systems 80, 67-77, 2015

[30] L. A. Zadeh, Fuzzy sets, Information and Control 8 (1965) 338-353

[31] W. Żakowski, Approximations in the space $(u, \pi)$, Demonstratio Mathematica 16 (1983) 761-769

[32] W. Zhu, Properties of the first type of covering-based rough sets, Sixth IEEE International Conference on Data Mining - Workshops IEEE. (2006).

[33] W. Zhu, F. Wang, On three types of covering based rough sets. IEEE Transactions on Knowledge and Data Engineering, No. 8 (2007). 